\title{
Structure-Function and Therapeutic Potential of Spider Venom-Derived Cysteine Knot Peptides Targeting Sodium Channels
}

\author{
Fernanda C. Cardoso* and Richard J. Lewis* \\ Centre for Pain Research, Institute for Molecular Bioscience, The University of Queensland, St Lucia, QLD, Australia
}

Spider venom-derived cysteine knot peptides are a mega-diverse class of molecules that exhibit unique pharmacological properties to modulate key membrane protein targets. Voltage-gated sodium channels ( $\mathrm{Nav}$ ) are often targeted by these peptides to allosterically promote opening or closing of the channel by binding to structural domains outside the channel pore. These effects can result in modified pain responses,

OPEN ACCESS

Edited by:

Jean-Francois Rolland, AXXAM, Italy

Reviewed by:

Roope Mannikko,

University College London,

United Kingdom Heike Wulff,

University of California, Davis, United States

*Correspondence:

Fernanda C. Cardoso f.caldascardoso@uq.edu.au

Richard J. Lewis

r.lewis@uq.edu.au;

r.lewis@imb.uq.edu.au

Specialty section:

This article was submitted to

Pharmacology of Ion Channels

and Channelopathies,

a section of the journal

Frontiers in Pharmacology

Received: 08 February 2019

Accepted: 25 March 2019

Published: 11 April 2019

Citation:

Cardoso FC and Lewis RJ (2019)

Structure-Function and Therapeutic Potential of Spider Venom-Derived Cysteine Knot Peptides Targeting

Sodium Channels.

Front. Pharmacol. 10:366. doi: 10.3389/fphar.2019.00366 muscle paralysis, cardiac arrest, priapism, and numbness. Although such effects are often deleterious, subtype selective spider venom peptides are showing potential to treat a range of neurological disorders, including chronic pain and epilepsy. This review examines the structure-activity relationships of cysteine knot peptides from spider venoms that modulate $\mathrm{Nav}$ and discusses their potential as leads to novel therapies for neurological disorders.

Keywords: spider venoms, ICK peptides, voltage-gated ion channels, structure-activity relationship, novel drugs

\section{INTRODUCTION}

Animal venoms are an extraordinary source of bio-active peptides that modulate membrane proteins to facilitate prey capture and defense. Venomous spiders, cone snails, fish, sea anemones, wasps, scorpions, snakes and dinoflagellates produce small molecules and/or peptides exhibiting pharmacological properties of singular value for the research in pharmacological tools and novel drugs (Ziegman and Alewood, 2015; Dongol et al., 2016; Cardoso and Lewis, 2017; Kessler et al., 2017; Abraham and Lewis, 2018; Cardoso et al., 2018). These toxins modulate a range of receptors and channels, including VGIC, TRP, muscarinic and nicotinic acetylcholine receptors (mAChR and nAChR), ASIC, NET and G protein coupled receptors (GPCRs). Based on the number of species and venom complexity, spider venoms provide a mega-diverse source of bioactive cysteine knot peptides, many of which modulate $\mathrm{Na}_{\mathrm{V}}$ with high potency and selectivity (Cardoso and Lewis, 2017).

Voltage-gated sodium channels ( $\left.\mathrm{Na}_{\mathrm{V}} 1.1-1.9\right)$ ), in particular, are key players in the transmission of electrical signals in excitable cells and also involved in the pathophysiology of neurological disorders, including poorly treated conditions as chronic pain and epilepsy (Luiz and Wood, 2016; Klein-Weigel et al., 2018; Sloan et al., 2018; Szepetowski, 2018). The Na $\mathrm{V}_{V}$ channels

Abbreviations: ASIC, acid sensing channels; Cav, voltage-gated calcium channel; CyA, $\beta$-cyanoalanine; DRG, dorsal root ganglion; ICK, inhibitory cysteine knot; $\mathrm{K}_{\mathrm{V}}$, voltage-gated potassium channel; mAChR, muscarinic acetylcholine receptor; nAchR, nicotinic acetylcholine receptor; $\mathrm{Na}$, voltage-gated sodium channels; $\mathrm{Na}_{V} \mathrm{PaS}$, insect $\mathrm{Na}_{V}$ channel; NET, norepinephrine transporter; Pra, propargylglycine; PyroE, pyroglutamate; SAR, structure-activity relationship; TRP, transient receptor potential channels; TTX-R, tetrodotoxin-resistant; TTX-S, tetrodotoxin-sensitive; VGIC, voltage-gated ion channels; VSD, voltage-sensor domain. 
$\alpha$-subunit comprises four domains (DI-DIV), each formed by six transmembrane segments (S1-S6), including $\mathrm{S} 4$ which contributes transmembrane voltage sensitivity, the tip of S5-S6 which contributes to sodium ion selectivity, and the intracellular loop connecting S6 of DIII and S1 of DIV which contributes to fast inactivation (Figure 1). The co-associated auxiliary $\beta$-subunits $(\beta 1-\beta 4)$ are positioned above the VSD explaining their ability to influence channel gating (Figures 1A,B). Interestingly, human genetic studies disclosed mutations in $\mathrm{Na}_{V} 1.7$ and $\mathrm{Na}_{V}$ 1.9 channels leading to congenital insensitivity to pain, a rare condition characterized by lack of physical pain (Cox et al., 2006; Phatarakijnirund et al., 2016), while gain-of-function mutations in $\mathrm{Na}_{V} 1.6, \mathrm{Na}_{V} 1.7, \mathrm{Na}_{V} 1.8$, and $\mathrm{Na}_{V} 1.9$ lead to painful neuropathies such as trigeminal neuralgia and erythromelalgia (Drenth and Waxman, 2007; Faber et al., 2012; Huang et al., 2014; Grasso et al., 2016). Genetic mutations in the Nav1.1 and $\mathrm{Na}_{V} 1.2$ channels result in functional defects linked to epileptic syndromes (Meisler and Kearney, 2005; Thompson et al., 2011). Furthermore, altered $\mathrm{Na}$ V channels function and expression are prominent in chronic inflammatory and neuropathic pains, with localization remodeling, altered expression and sensitization often observed in the subtypes $\mathrm{Na}_{V}$ 1.3, $\mathrm{Na}_{V}$ 1.6, $\mathrm{Na}_{V}$ 1.7, $\mathrm{Na}_{V} 1.8$, and $\mathrm{Na}_{V} 1.9$ (Cardoso and Lewis, 2017). $\mathrm{Na}_{V} 1.4$ and $\mathrm{Na}_{V} 1.5$ have restricted expression in the skeletal and cardiac muscle, respectively, which are important off-target pharmacologies to be considered when developing $\mathrm{Na}_{V}$ channel therapeutics.

The role of $\mathrm{Na}_{V}$ channels in both health and pathological pain have been further elucidated with the support of potent and selective $\mathrm{Na}_{V}$ channels modulators isolated from spider venoms. By using a subtype selective $\mathrm{Na}_{V} 1.1$ activator isolated from the tarantula Heteroscodra maculata, the key role played by $\mathrm{Na}_{V} 1.1$ in physiological mechanical pain (Osteen et al., 2016) and chronic visceral pain (Salvatierra et al., 2018) was established. More interestingly, pain relief is achieved in pre-clinical models of inflammatory and neuropathic pain administrated with $\mathrm{Na}_{\mathrm{V}}$ channels inhibitors isolated from other spiders venoms, as for the ICK peptides ProTx-II (Tanaka et al., 2015; Flinspach et al., 2017), HnTX-IV (Liu et al., 2014a), Hl1a (Meng et al., 2016), HwTx-IV (Liu et al., 2014b), and Pn3a (Deuis et al., 2017).

The unique properties of spider ICK peptides in modulating ion channels give rise to opportunities for developing better and safer therapies targeting $\mathrm{Na}_{V}$ channels. This, in association to the current need for effective drugs to treat challenging neurological disorders and to overcome severe side-effects by opioid analgesic drugs foster the use of these bio-active spider peptides in therapeutics development. In this review, we examine recent advances in the SAR of cysteine knot peptides from spider venoms that inhibit $\mathrm{Na}_{\mathrm{V}}$ and discuss their potential for the development of novel therapies.

\section{STRUCTURE-ACTIVITY RELATIONSHIPS OF SPIDER ICK PEPTIDES}

Spiders are the largest group of venomous animals, with more the 40,000 species described to date (Platnick, 2014). Their venoms are rich in peptides that inhibit or activate $\mathrm{Na}_{\mathrm{V}}$ channels by binding to domains outside the channel pore to allosterically promote opening or closing of the channel (Figures 1A-C) (Cardoso and Lewis, 2017). These binding sites include the VSD associated with domains II and IV that bind site 4 and $3 \mathrm{Na}_{\mathrm{V}}$ channel toxins, respectively. Interestingly, these peptides have a conserved ICK scaffold that confers high stability and resistance to high temperatures, low $\mathrm{pH}$ and digestion by proteases. A typical spider peptide ICK scaffold, with few exceptions, comprises three disulphide bridges $\mathrm{C} 1-\mathrm{C} 4, \mathrm{C} 2-\mathrm{C} 5$, and $\mathrm{C} 3-$ C6 that fold these peptides into a globular structure with four distinct loops and an extended C-terminal tail (Figure 1D). These peptides were classified into distinct families of $\mathrm{Na}_{\mathrm{V}}$ modulators named NaSpTx 1-12 based on their amino acids sequence and cysteine position (Klint et al., 2012), with extensive SAR studies reported for NaSpTx 1 and 3, and to a lesser extent for NaSpTx 7. Modern high throughput screening technologies using fluorescence-imaging and automated patch-clamp cellbased assays have facilitated the identification of peptides that display high potency and selectivity to modulate $\mathrm{Na}_{V}$ subtypes (Cardoso et al., 2015; Klint et al., 2015b). These peptides have been the focus of studies to unravel their pharmacological properties and potential for the development of novel and more effective drugs, studies which have been considerably advanced through investigations of the SAR of spider ICK peptides over $\mathrm{Na}_{V}$ channel subtypes. By using state-of-art methods for peptide production and detailed pharmacology characterization through patch-clamp electrophysiology in primary isolated neurons (e.g., DRG) or mammalian cells expressing $\mathrm{Na}_{\mathrm{V}}$ channels subtypes, and $\mathrm{X}$-ray and nuclear magnetic resonance (NMR) for determination of three-dimensional structure, these SAR studies have unraveled key features associated to modulation $\mathrm{Na}_{V}$ channels. Our current understanding of the SAR of each of the main ICK family peptides at $\mathrm{Na}_{\mathrm{V}}$ channels are outlined below.

\section{NaSpTx 1}

\section{GpTx-1}

The ICK peptide GpTx-1 ( $\mu / \omega$-TRTX-Gr2a) isolated from the tarantula Grammostola rosea was first described as a nanomolar inhibitor of $\mathrm{Ca}_{\mathrm{V}}$ (Ono et al., 2011) and later as a nanomolar $\mathrm{Na}_{\mathrm{V}}$ inhibitor from Grammostola porter (Murray et al., 2015). Its high potency for the sodium channel subtype $\mathrm{Na}_{\mathrm{V}} 1.7$ ( $\mathrm{IC}_{50}$ of $\left.10 \mathrm{nM}\right)$ made it an attractive lead for SAR studies (Murray et al., 2015, 2016) (Table 1). Alanine scanning revealed the residues W29, K31 and F34 are essential for the $\mathrm{Na}_{\mathrm{V}} 1.7$ inhibition (Murray et al., 2015). In its native form, GpTx-1 is 20-fold and 1000fold selective over $\mathrm{Na}_{V} 1.4$ and $\mathrm{Na}_{V} 1.5$, respectively, with the F5A mutant enhancing to 300 -fold selectivity over $\mathrm{Na}_{V} 1.4$. In the same study, additional positional substitutions using natural and non-natural amino acids other than alanine in the positions 5, 6, 26 , and 28 put forward the rational design of an optimized GpTx1 containing the substitutions F5A, M6F, T26L, K28R. This new GpTx-1 analog displayed 6-fold enhanced potency for $\mathrm{Na}_{\mathrm{V}} 1.7$ and 1000-fold selectivity over $\mathrm{Na}_{\mathrm{V}} 1.4$ and $\mathrm{Na}_{\mathrm{V}} 1.5$.

Later, another positional scan using glutamic acid, arginine, lysin, tryptophan and 1-naphthylalanine substitutions supported 

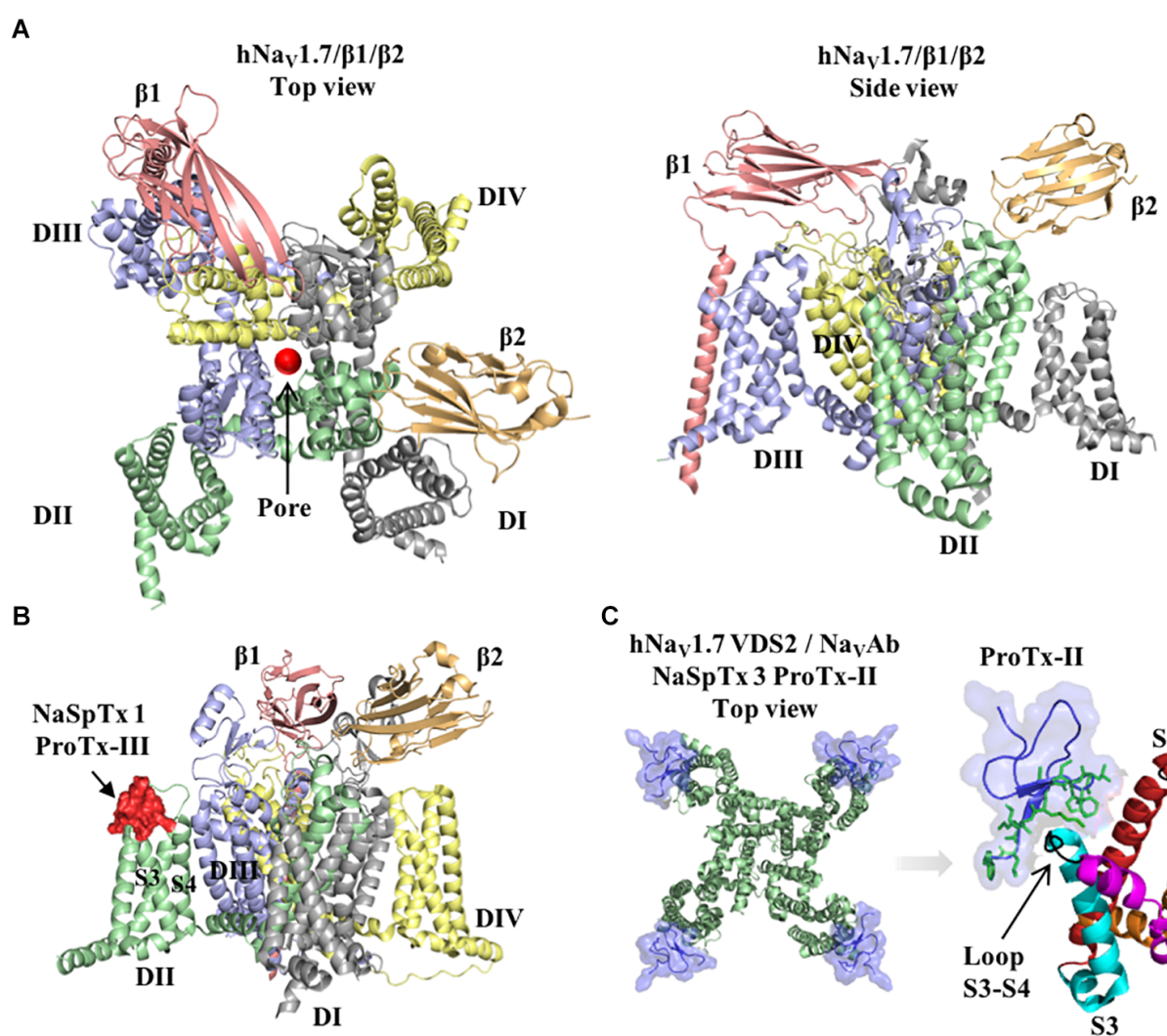

C

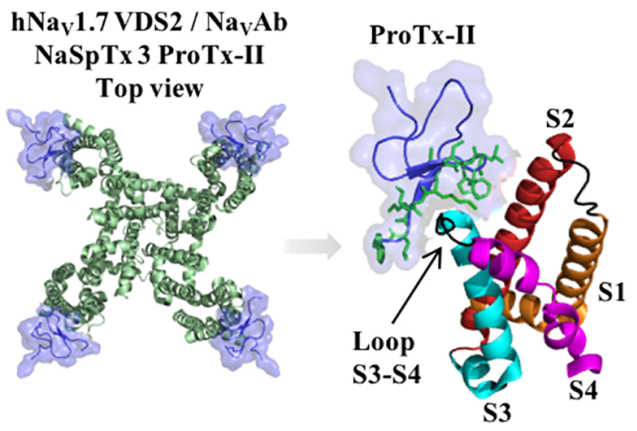

D
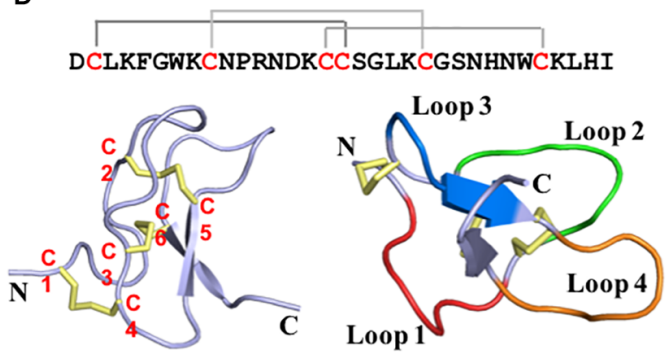

FIGURE 1 | Structure of the voltage-gated sodium channel and the inhibitory cysteine knot (ICK) spider peptide. (A) Three-dimensional structure of the human Nav1.7 in the presence of the auxiliary subunits $\beta 1$ and $\beta 2$ determined by cryo-EM (PDB 6J8J) (Shen et al., 2019). Top and side views are presented. The domain I (DI) is colored in gray, domain II (DII) is colored in green, domain III (DIII) is colored in blue and domain IV (DIV) is colored in yellow. The auxiliary subunits $\beta 1$ and $\beta 2$ are colored in salmon and orange, respectively. (B) Representative binding site of a NaSpTx 1 in the DII S3-S4 loop of the hNav1.7 channel (PDB 6J8J) (Shen et al., 2019). Domains and auxiliary subunits are colored as in (A), and the NaSpTx1 peptide ProTx-III (PDB 2MXM) (Cardoso et al., 2015) is colored in red. (C) Detailed binding site of the NaSpTx 3 ProTx-II over the hNav1.7 voltage-sensor domain 2 (VSD2)-NavAb chimeric channel (PBD 6N4I) (Xu et al., 2019). Top view of the hNav1.7 VSD2-NavAb chimeric channel colored in green showing ProTx-II colored in blue bound to the voltage sensor domain 2 in close proximity with the S3-S4 loop. In the structure on the left, the segments S1 to S4 are colored in orange, red, cyan and magenta, respectively. The loops S1-S2 and S3-S4 are colored in black and the residues in the loop 4 and C-terminal of ProTx-II are represented by green sticks. (D) Typical structure of an inhibitory cysteine knot (ICK) peptide from spider. Primary and three-dimensional structure of ProTx-III (PDB 2MXM) (Cardoso et al., 2015) showing cysteine connectivity (C1 - C4, C2 - C5, and C3 - C6) and loops 1-4 colored in red, green, blue and orange, respectively.

previous alanine scanning observations and revealed a number of new positions in GpTx-1 essential for the $\mathrm{Na}_{\mathrm{V}} 1.7$ activity, all together these key residues are F5, M6, S24, H27, W29, K31, Y32, and F34 (Murray et al., 2016). Contrary to alanine substitutions, most of the substitutions with 1-naphthylalanine didn't produce folded peptides, with few similar cases for substitutions with tryptophan, followed by lysine, arginine and glutamic acid. Furthermore, the importance of S24 was observed only in the
S24E mutant, being all other substitutions at position 24 unable to produce folded peptides. In the three-dimensional structure of GpTx-1, these identified active residues form a cluster on the surface allowing a direct interaction with $\mathrm{Na}_{\mathrm{V}} 1.7$ (Murray et al., 2016). These potential interactions were further investigated using a homology model of the hNav 1.7 channel docked with the NMR structure of GpTx-1, which predicted interactions of the residues F5 and $\mathrm{K} 31$ of GpTx-1 with $\mathrm{I} 767$ and E811 located in 
the domain II voltage sensor region, respectively. Other potential electrostatic interactions were predicted between R25 and R7 of GpTx-1 and E759 and E818 of the ion channel, respectively. Overall, the optimization of GpTx-1 toward an increase in potency for $\mathrm{Na}_{V} 1.7$ and selectivity over $\mathrm{Na}_{V} 1.4$ and $\mathrm{Na}_{V} 1.5$ was possible with the addition of a hydrophobic aromatic residue at position 6 (substitution M6F), and a hydrophobic residue at position 26 (substitution T26L). A summary of the SAR for GpTx-1 is shown in Table 1.

\section{HwTx-IV}

Huwentoxin-IV ( $\mu$-TRTX-Hs2a), isolated from the venom of the Chinese bird spider Selenocosmia huwena, was first identified as a potent ( $\mathrm{IC}_{50}$ of $30 \mathrm{nM}$ ) Nav inhibitor of TTX-S currents in DRG neurons (Peng et al., 2002). This inhibitor has been the subject of extensive SAR studies (Table 1 and Figure 2). Initial SAR observations for HwTx-IV described a naturally occurring toxin variant with an $\mathrm{N}$-terminal pyroglutamate instead of glutamate that had unchanged potency despite its irreversibility at strong depolarizing potentials (e.g., $+200 \mathrm{mV}$ ) in DRG neurons and in $\mathrm{Na}_{V} 1.7$ expressed in HEK293 cells (Rong et al., 2013). More detailed SAR studies of HwTx-IV revealed that C-terminal mutations T28D, R29A or Q34D reduced Nav potency in DRG neurons (Deng et al., 2013), while an alanine scan of HwTXIV additionally revealed W30 and K32 also critical for high affinity interactions at $\mathrm{Na}_{V} 1.2$ and $\mathrm{Na}_{V} 1.7$ (Minassian et al., 2013) (Figure 2A). In this latter study, molecular dynamics simulations revealed substitutions displaying loss of potency for $\mathrm{Na}_{V} 1.2$ and $\mathrm{Na}_{\mathrm{V}} 1.7$ also led to an increase in the flexibility of the loops 2 (substitutions P11A and D14A), in loop 3 (substitutions F6A, L22A, W30A, and Y33A) and in loop 4 (substitutions R26A and K27A), while the substitutions S25A, K32A, and I35A reduced HwTx-IV potency for $\mathrm{Na}_{V} 1.2$ and Nav1.7 without affecting its structure and loops flexibility (Minassian et al., 2013).

Further alanine scanning of HwTx-IV identified E1, E4, F6, and Y33 as important contributors for Nav1.7 affinity (Revell et al., 2013). These findings afforded the rational design and optimization of HwTx-IV with increased potency for $\mathrm{Na}_{V} 1.7$ and maintaining low affinity for the off-target $\mathrm{Na}_{V} 1.5$ (Figure 3). Among these new HwTx-IV analogs, the mutant HwTx-IVG1/G4/W33 demonstrated the highest increase in activity, with 42-fold enhanced potency for $\mathrm{Na}_{\mathrm{V}} 1.7$ inhibition, followed by HwTx-G1/G4 and HwTx-IV-A1/A4/W33 (Revell et al., 2013). More recently, the activity of HwTx-IV-G1/G4/W33 was tested over other members of the $\mathrm{Na}_{V}$ family, showing an increase in inhibition of 12-fold for $\mathrm{Na}_{V} 1.2$ and 47-fold for $\mathrm{Na}_{V} 1.3$ compared to wild-type HwTx-IV, but maintained the low potency for the off-target $\mathrm{Na}_{V} 1.4$ and $\mathrm{Na}_{V} 1.5$ (Xiao et al., 2008a; Rahnama et al., 2017). Altogether, the residues F6, P11, D14, L22, T28, R29, W30, K32, Y33, and Q34, when mutated, led to a loss of affinity of HwTx-IV for Nav channels (Table 1 and Figure 3A). Furthermore, the optimization of HwTx-IV activity for $\mathrm{Na}_{V} 1.7$ was possible with the removal of acid negative residues at the $\mathrm{N}$-terminal (substitutions E1 to A or G), and increase in hydrophobicity at the C-terminal (substitution Y33W) (Table $\mathbf{1}$ and Figure 3A). These produced a new surface in the HwTx-IV three-dimensional structure with increased polarity and hydrophobicity.

\section{CcoTx1}

CcoTx1 ( $\beta$-TRTX-Cm1a) is a potent $\mathrm{Na}_{\mathrm{V}}$ inhibitor isolated from the tarantula Ceratogyrus cornuatus (Bosmans et al., 2006). It has strong preference for the subtype $\mathrm{Na}_{V} 1.2$ and lower affinity for the off-targets $\mathrm{Na}_{V} 1.4$ and $\mathrm{Na}_{V} 1.5$ (Table 1). The SAR of CcoTx1 was investigated using a combination of direct evolution, saturation mutagenesis, chemical modifications and rational drug design to unravel key residues involved in potency and selectivity of this peptide over the $\mathrm{Na}_{V}$ family (Shcherbatko et al., 2016). Using direct evolution, CcoTx1 was optimized to improve its potency for the subtype $\mathrm{Na}_{V} 1.7$, and off-target selectivity for $\mathrm{Na}_{V} 1.2$ and $\mathrm{Na}_{V} 1.6$, producing the variant named 2670. This mutant contained the substitutions W5M, K12E, N19R, Y20L, T21V, R25S, D26H, Y31W, and D32K (Table 1 and Figure 3B).

Further SAR investigations aiming to improve selectivity and potency for $\mathrm{Na}_{\mathrm{V}} 1.7$ were performed using saturation mutagenesis, revealing that substitutions at the $\mathrm{N}$ - and C-terminal regions, along with positions 20 and 21 of 2670 , were not well tolerated. Combining this SAR information, the $\mathrm{Na}_{\mathrm{V}} 1.7$ pharmacophore of 2670 was defined by the hydrophobic residues M5, F6, W28, W31, and L33, and polar or positively charged residues $\mathrm{R} 19, \mathrm{H} 26, \mathrm{~K} 30$, and K32. Saturation mutagenesis substitutions that conferred improved selectivity over off-targets and didn't alter the $\mathrm{Na}_{V} 1.7$ inhibitory potency were K18Y, $\mathrm{R} 24 \mathrm{~K}$, and R27N. Still using this approach, a new mutant was identified with one single substitution D1I that conferred improved activity and selectivity (Table 1). C-terminal amidation of 2670 (2670a) and D1I (D1Ia) improved potency for Na 1.7 which was accompanied by an increase in potency for $\mathrm{Na}_{V} 1.6$, but not for $\mathrm{Na}_{\mathrm{V}}$ 1.2. In addition, a D1Ia variant containing a terminal pyroglutamate (D1Za) improved binding to Nav 1.7 by $27 \%$ at strong depolarizing potentials, and maintained the same potency as D1Ia. This observation resembles the SAR properties of HwTxIV (Rong et al., 2013). Overall, the optimization of CcoTx-1 activity toward $\mathrm{Na}_{\mathrm{V}} 1.7$ was possible with the replacement of acid negative residues at both $\mathrm{N}$ - and $\mathrm{C}$-terminal to residues with increased hydrophobicity and positive charges, and with modifications toward the C-terminal that included substitutions for aromatic residues $\mathrm{H}$ and $\mathrm{W}$. This led to a considerable change in the CcoTx-1 surface to include more hydrophobic and positively charged residues (Figure 3B).

\section{Hainantoxin}

Hainantoxins (HNTXs) are ICK peptides comprised in the venom of the Chinese bird spider Selenocosmia hainana. Among these, the HNTX-I ( $\mu$-TRTX-Hhn2b) has weak to no activity over $\mathrm{Na}_{V}$ channels. SAR studies to restore the $\mathrm{Na}_{V}$ inhibitory activity of HNTX-I were performed using rational design based on other members of the NaSpTx 1 (Klint et al., 2015a; Zhang et al., 2018b) (Table $\mathbf{1}$ and Figure 3C). The substitutions G6W/N23S/W28F and G6W/N23S produced analogs with IC $_{50}$ values of 1 and $0.44 \mu \mathrm{M}$ for $\mathrm{Na}_{\mathrm{V}} 1.7$, respectively (Klint et al., 2015a). Later, the analogs N23S/D26H, N23S/D26H/L32W and E1G/N23S/D26H/L32W showed $\mathrm{IC}_{50}$ values of 79, 71, and $36 \mathrm{nM}$ 
TABLE 1 | Structure-activity relationship of spider ICK peptides and Nav channels.

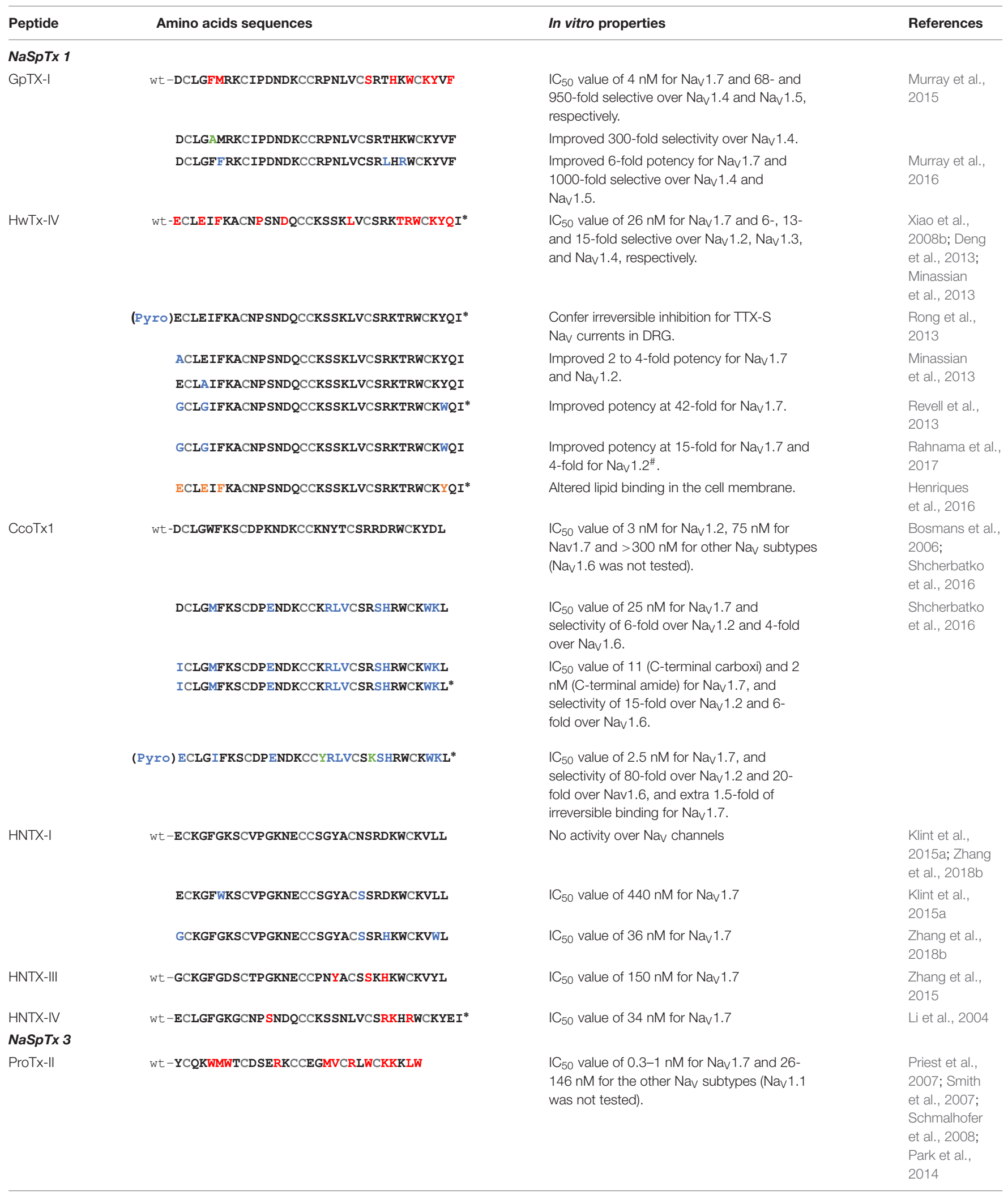


TABLE 1 | Continued

\begin{tabular}{|c|c|c|c|}
\hline Peptide & Amino acids sequences & In vitro properties & References \\
\hline & YCQKWMWTCDSERKCCEGMVCRLWCKKKLW-NHCH 3 & $\begin{array}{l}\mathrm{IC}_{50} \text { value of } 42 \text { pMol for Nav1.7, } \\
\text { selectivity of } 83 \text {-fold over Nav } 1.2 \text {. }\end{array}$ & $\begin{array}{l}\text { Park et al., } \\
2014\end{array}$ \\
\hline & GPYCQKWMQTCDSERKCCEGMVCRLWCKKKLL & Improved selectivity over Nav1.4 and Nav1.5. & $\begin{array}{l}\text { Flinspach et al., } \\
2017\end{array}$ \\
\hline & YCQKWMWTCDSERKCCEGMVCRLWCKKKLW & Altered lipid binding in the cell membrane. & $\begin{array}{l}\text { Agwa et al., } \\
2017\end{array}$ \\
\hline \multirow[t]{4}{*}{ JZTX-V } & wt-YCQKWMWTCDSKRACCEGLRCKLWCRKII* & $\begin{array}{l}\mathrm{IC}_{50} \text { value of } 0.6 \mathrm{nM} \text { for } \mathrm{Nav} 1.7 \text {, and } \\
\text { selectivity of } 4 \text { - and } 4000 \text {-fold over Nav } 1.4 \\
\text { and Nav1.5, respectively. }\end{array}$ & $\begin{array}{l}\text { Moyer et al., } \\
2018\end{array}$ \\
\hline & YCQKWMWTCDSKRACCEGLRCKLWCRKEI* & $\begin{array}{l}\text { Improved selectivity over Nav } 1.4 \text { to } 500 \text { - } \\
\text { fold (AM-8145). }\end{array}$ & \\
\hline & (Pra) YCQKWMWTCDSKRACCEGLRCKLWCRKEI* & $\begin{array}{l}\text { Improved selectivity to } 300 \text { - and } 6000 \text {-fold } \\
\text { over Nav } 1.4 \text { and Nav1.5, respectively. }\end{array}$ & \\
\hline & $\begin{array}{c}\text { (CYA) YCQKWMWTCDSKRACCEGLRCKLWCRKEI* } \\
\text { (Pra) }\end{array}$ & $\begin{array}{l}\text { Improved selectivity to } 128 \text { - and } 1200 \text {-fold } \\
\text { over Nav } 1.4 \text { and Nav } 1.5 \text {, respectively. }\end{array}$ & \\
\hline \multicolumn{4}{|c|}{ NaSpTx 7} \\
\hline \multirow[t]{2}{*}{ JZTX-III } & wt-DGECGGFWWKCGRGKPPCCKGYACSKTWGWCAVEAP & $\begin{array}{l}\mathrm{IC}_{50} \text { value of } 348 \mathrm{nM} \text { for Nav1.5, not active } \\
\text { over other Nav subtypes }\end{array}$ & $\begin{array}{l}\text { Rong et al., } \\
2011\end{array}$ \\
\hline & DGECGGFWWKCGEGKPPCCKGYACSKTWGWCAVEAP & Improved potency by 11 -fold for Nav1.5. & \\
\hline
\end{tabular}

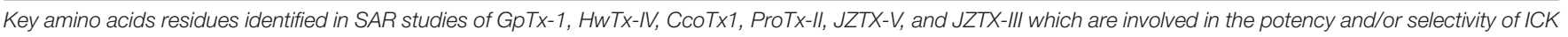

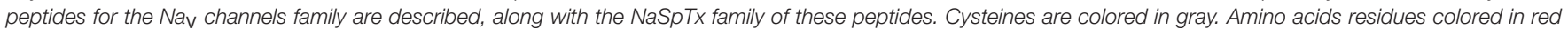

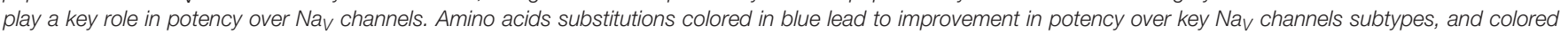

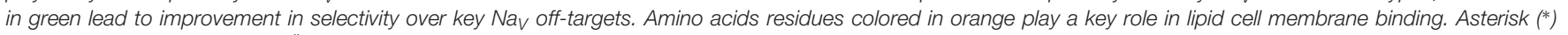
denotes C-terminal amidation. " Compared to Minassian et al. (2013). wt, wild-type.

for $\mathrm{Na}_{V} 1.7$, respectively. In this study, the motif $\mathrm{X}_{1} \mathrm{X}_{2} \mathrm{SWCKX}_{3}$ was identified as critical for the inhibitory activity for $\mathrm{Na}_{\mathrm{V}} 1.7$. Altogether, the HNTX-I activity for $\mathrm{Na}_{V} 1.7$ was restored by removal of acid negative residues at both $\mathrm{N}$ - and C-terminal, and the addition of residues with increased hydrophobicity and positive charges, including the aromatic residues $\mathrm{H}$ and $\mathrm{W}$.

Another hainantoxin, HNTX-III ( $\mu$-TRTX-Hhn2a), is an inhibitor of $\mathrm{Na}_{\mathrm{V}} 1.7$ with $\mathrm{IC}_{50}$ value of $232 \mathrm{nM}$ (Liu et al., 2013). The SAR of this peptide was performed consequent to the discovery of several isoforms variants present in the transcriptome of the venom gland of this spider (Zhang et al., 2015) (Table 1). The pharmacological properties of these variants were tested, revealing that the substitutions Y20H, S24N, H26D and $\mathrm{Y} 20 \mathrm{H} / \mathrm{S} 24 \mathrm{~N}$ were all detrimental to the NaV1.7 activity (Figure 2B). The last hainantoxin discussed is HNTX-IV ( $\mu$-TRTX-Hhn1b), a potent inhibitor of TTX-S currents in DRG neurons ( $\mathrm{IC}_{50}$ of $34 \mathrm{nM}$; Li et al., 2004). In this study, the SAR of HNTX-IV revealed K27 and R29 residues positions involved in the $\mathrm{Na}_{V}$ activity (Table $\mathbf{1}$ and Figure 2C). More specifically, the mutants S12A and R26A produced $\mathrm{IC}_{50}$ values of 58 and $96 \mathrm{nM}$, respectively, while $\mathrm{K} 27 \mathrm{~A}$ and $\mathrm{R} 29 \mathrm{~A}$ produced $\mathrm{IC}_{50}$ values of 3.2 and $7 \mu \mathrm{M}$, respectively. This work reinforces the essential role of positively charged residues located in the surface of spider ICK peptides for $\mathrm{Na}_{\mathrm{V}} 1.7$ inhibition.

\section{NaSpTx 3}

\section{ProTx-II}

ProTx-II $\left(\beta / \omega\right.$-TRTX-Tp2a) is amongst the most potent $\mathrm{Na}_{V}$ inhibitors described to date, with reported $\mathrm{IC}_{50}$ value of $0.3 \mathrm{nM}$ for inhibition of $\mathrm{Na}_{V} 1.7$ (Schmalhofer et al., 2008). This toxin was isolated from the spider Thrixopelma pruriens using rNav1.8 assay guided fraction that evaluated the inhibitory properties for the $\mathrm{Na}_{\mathrm{V}} 1.8$ channel of individual venom fractions separated by cation exchange liquid chromatography (Middleton et al., 2002). Despite of its exquisite inhibitory potency for $\mathrm{Na}_{V} 1.7$, it also potently inhibits other members of the $\mathrm{Na}_{V}$ family, including the off-targets $\mathrm{Na}_{V} 1.4$ and $\mathrm{Na}_{V} 1.5$. The SAR of ProTx-II was initially investigated over the $\mathrm{Na}_{V} 1.5$ channel (Table 1 and Figure 2D). Analogs of ProTx-II produced by recombinant expression and chemical synthesis containing alanine or glutamine substitutions revealed a peptide active face composed of hydrophobic and cationic residues (Smith et al., 2007). More specifically, ten of these analogs showed losses in potency from 10- to 125-fold, with major losses associated with W5A and K26A substitutions. The positively charged residues substitutions K27Q, R13Q, and R22A led a significant loss in potency, while neutralization of negatively charged residues didn't affect the $\mathrm{Na}_{V} 1.5$ inhibition. Furthermore, the substitutions M6A, W7A, M19L, V20A, W24L, L29A, and W30A each produced a $>10$-fold loss in potency. Similarly, another SAR study of ProTx-II identified the residues in the hydrophobic face essential for Nav1.5 activity, including W5, M6, W7, W24, while residues identified as not critical for inhibitory activity included Y1, Q3, T8, N10, S11, E12, E17, and L23 (Priest et al., 2007).

SAR of ProTx-II has also been investigated over the channels $\mathrm{Na}_{V} 1.2$ and $\mathrm{Na}_{V} 1.7$ (Park et al., 2014) (Table 1 and Figure 2D). In this study, besides the identification of residues mutations detrimental to $\mathrm{Na}_{\mathrm{V}}$ activity such as substitution of C-terminal KLW to II, an optimized analog ProTx-II- $\mathrm{NHCH}_{3}$ with $\sim 23$-fold greater potency for $\mathrm{Na}_{V} 1.7$ was identified. In this same work, the 

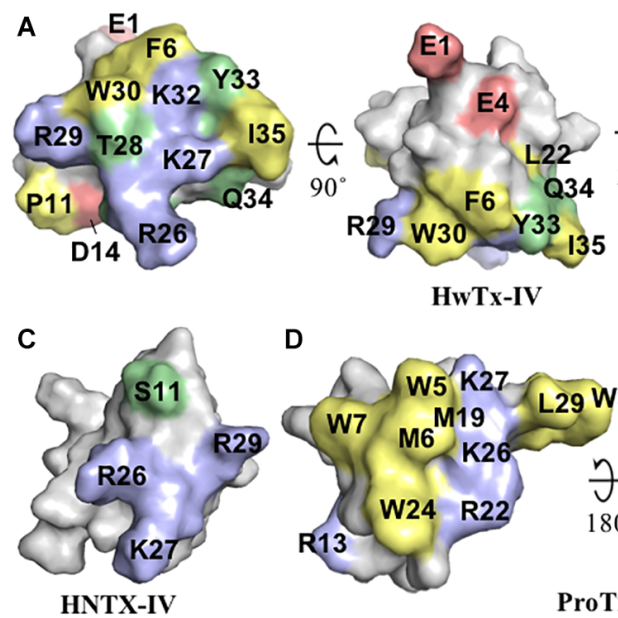

D

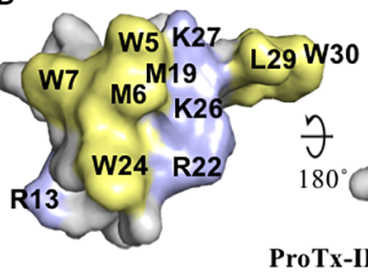

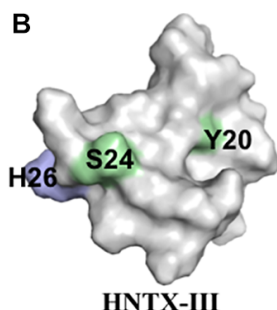

$E$

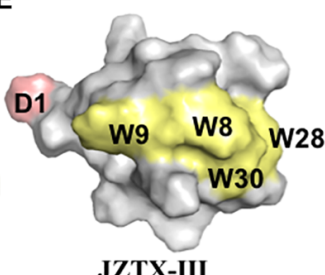

$\mathbf{F}$
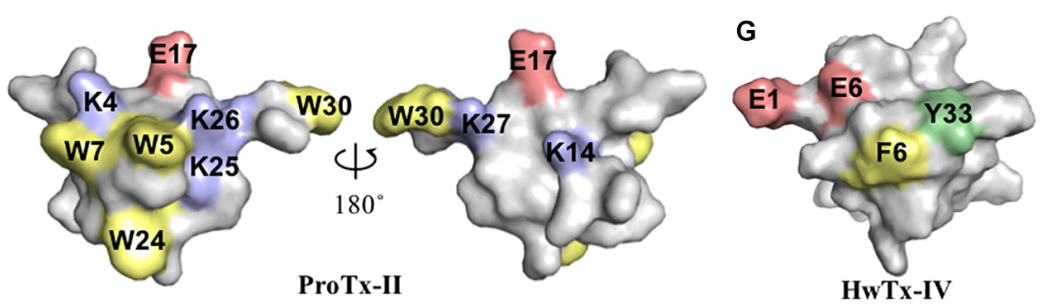

FIGURE 2 | Three-dimensional structure of spider ICK peptides displaying key residues involved in the inhibition of Nav channels and cell membrane binding. (A) Three-dimensional structure of HwTX-IV determined by NMR (PBD 2m4x) (Minassian et al., 2013), (B) HNTX-III determined by NMR (PBD 2jtb), (C) HNTX-IV determined by NMR (PBD 1niy) (Li et al., 2004), (D) ProTx-II determined by X-Ray (PBD 5o0u) (Wright et al., 2017), and (E) JZTX-III determined by NMR (PBD 2i1t). The labeled amino acids residues have key role in potency over Nav channels and lead to loss in activity as described in the text and Table 1. (F) Structure of ProTx-II determined by X-Ray (PDB 5o0u) (Wright et al., 2017) and (G) Structure of HwTx-IV (PDB 2m4x) (Minassian et al., 2013) determined by NMR. The labeled amino acids residues have key role in cell membrane binding as described in the text and Table 1. Amino acids residues are colored as follow: yellow for hydrophobic, red for acid, blue for basic and green for neutral. All three-dimensional structures were prepared in PyMOL (DeLano, 2002).

ICK peptide Phrixotoxin I, also known as PaTx I, isolated from the tarantula Phrixotrichus auratus, and a potent $\mathrm{K}_{\mathrm{V}}$ inhibitor with $\mathrm{IC}_{50}$ value of $28 \mathrm{nM}$ was submitted to a SAR study over $\mathrm{Na}_{V}$ channels (Diochot et al., 1999). It differs from ProTx-II by only 4 amino acids residues, and has moderate potency for $\mathrm{Na}_{V}$ channels, with $\mathrm{IC}_{50}$ value of $423 \mathrm{nM}$ for $\mathrm{Na}_{V} 1.7$, and no activity for $\mathrm{Na}_{\mathrm{V}} 1.2$ (Park et al., 2014). These naturally occurring differences were useful for the understand of the ProTx-II SAR over $\mathrm{Na}_{V}$ channels. The PaTx I C-terminal substitutions I28K and I29K, and addition of L30 and W31 (actual ProTx-II C-terminal) lead to an increase of 85 -fold in the Nav1.7 potency, and an $\mathrm{IC}_{50}$ value of $45 \mathrm{nM}$ for $\mathrm{Na}_{\mathrm{V}}$ 1.2. This work unraveled a key role of the C-terminal for activity of NaSpTx 3 peptides, and describes interesting approaches for the improvement of $\mathrm{Na}$ activity through C-terminal modifications.

More recently, a detailed SAR of ProTx-II over Nav channels was investigated by 1500 toxin-derived peptides to identify a double mutant with extended $\mathrm{N}$-terminal that maintained the inhibitory properties for $\mathrm{Na}_{V} 1.7$ but lost affinity for $\mathrm{Na}_{V} 1.4$ and Na 1.5 (Flinspach et al., 2017) (Table 1 and Figure 3D). This mutant, named JNJ63955918, contained additional G-2 and P1 at the N-terminal and the substitutions W7Q and W30L. The interactions of ProTx-II with lipid membranes as well as with the hNav1.7 channels were investigated, revealing ProTx-II does interact with the cell membrane as part of its strategy to inhibit the $\mathrm{Na}_{V} 1.7$ channel (Henriques et al., 2016) (Figure 3). In this study, substitutions of residues $\mathrm{K}$ to $\mathrm{R}$ and $\mathrm{W}$ to $\mathrm{Y}$ lead to a reduction in the potency of ProTx-II for hNa 1.7 . The ProTxII lipid interactions will be discussed in more details later in this review. Altogether, the residues W5, M6, W7, R13, M19, V20, R22, W24, K26, K27, L29, and W30, when mutated, led to a loss of affinity of ProTx-II for Nav channels (Table 1 and Figure 2D). Interestingly, the optimization of ProTx-II toward a potent and selective $\mathrm{Na}_{V} 1.7$ inhibitor was possible through the extension of the N-terminal with neutral and positively charged residues, and introduction of a positively charged group in the C-terminal.

\section{JZTX-V}

Isolated from the tarantula Chilobrachys jingzhao, JZTX-V $\left(\beta\right.$-TRTX-Cg2a) is a potent $\mathrm{Na}_{\mathrm{V}} 1.7$ inhibitor with $\mathrm{IC}_{50}$ value of $0.6 \mathrm{nM}$ (Moyer et al., 2018). The SAR of JZTX-V over the subtypes $\mathrm{Na}_{V} 1.4, \mathrm{Na}_{V} 1.5$, and $\mathrm{Na}_{V} 1.7$ was investigated using alanine or glutamic acid positional scanning to unravel key residues involved in $\mathrm{Na}_{\mathrm{V}}$ inhibition, and to produce new mutants with improved potency and selectivity (Table 1 and Figure 3E). Substitutions that contribute to loss of the $\mathrm{Na}_{V} 1.7$ inhibitory activity observed by alanine substitutions were W5, L19, W24, and R26, and by glutamic acid substitutions were M6, T8, D10, 

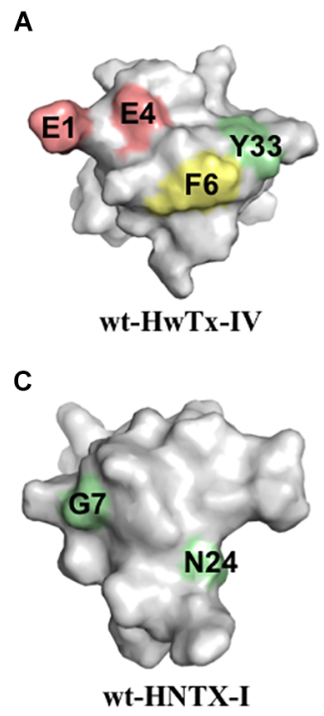

Pra-1 JZTX-V

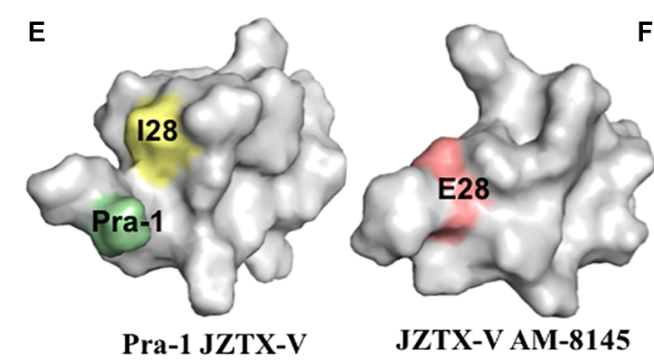

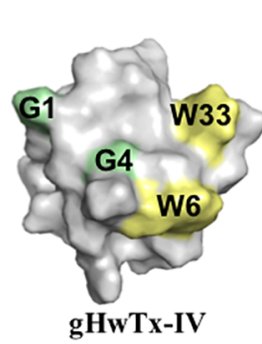
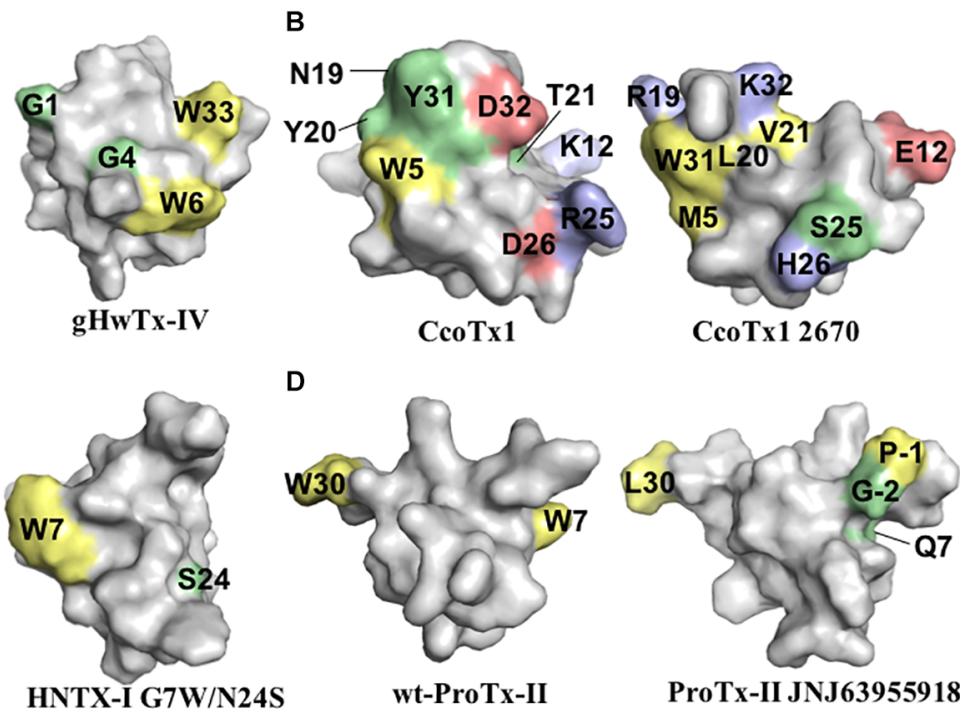

D
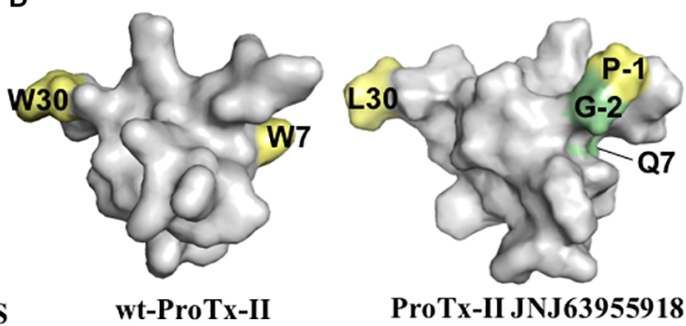

ProTx-II JNJ63955918

$\mathbf{F}$

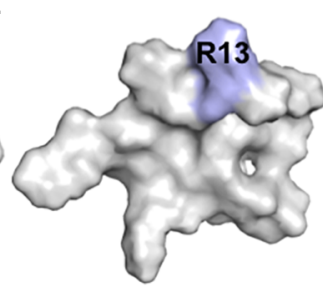

wt-JZTX-III

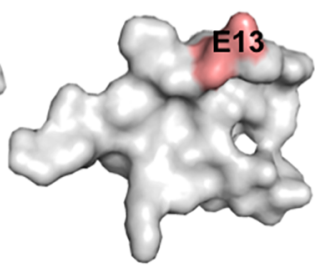

JZTX-III R13E

FIGURE 3 | Three-dimensional structure of spider ICK peptides displaying key residues involved in the enhancement of activity for Nav channels, or enhancement of selectivity for Nav channels off-targets. (A) Structures of HwTx-IV (PDB 2m4x) (Minassian et al., 2013) and gHwTx-IV (PDB 5tr) (Agwa et al., 2017) determined by NMR. (B) Structures of CcoTx1 determined by NMR (PDB 6br0) (Agwa et al., 2018) and the derived analog 2670 determined by X-ray (PDB 5epm) (Shcherbatko et al., 2016). (C) Structures of HNTX-I (PDB 2mqf) and the derived analog G7W/N24S (PDB 2mxo) determined by NMR (Klint et al., 2015a). (D) Structures of ProTx-II determined by X-Ray (PDB 5oOu) (Wright et al., 2017) and derived analog JNJ63955918 determined by NMR (PDB 5tcz) (Flinspach et al., 2017). (E) Structure of analogs Pra-1 JZTX-V (PDB 6chc) and AM-8145 (6cgw) determined by NMR (Moyer et al., 2018). (F) Structure of JZTX-III determined by NMR (PDB 2i1t) (Liao et al., 2006) and modeling of the derived analog R13A using SWISS-MODEL (Arnold et al., 2006). Amino acids residues are colored as follow: yellow for hydrophobic, red for acid, blue for basic and green for neutral. Amino acids substitutions or additions labeled in the respective analogs are involved in the improvement of Nav inhibitory activity or selectivity as described in the text and Table 1. All three-dimensional structures alignments were prepared in PyMOL (DeLano, 2002).

R13, and L23. Interestingly, a key substitution that improved off-target selectivity of JZTX-V over $\mathrm{Na}_{\mathrm{V}} 1.4$ was I28E. This mutation was able to induce a change in the conformation of JZTX-V to produce 500-fold selectivity and maintain the potency for $\mathrm{Na}_{V} 1.7$.

In the same study, further investigation of the SAR of JZTX-V was performed through the introduction of side-chains containing residues such as Pra and $\beta$-cyanoalanine (CyA). Positions showing minimum involvement in $\mathrm{Na}_{V} 1.7$ inhibitory activity disclosed by the glutamic acid scanning were Y1, S11, A14, and E17, and therefore selected to test this approach. The addition of Pra at the N-terminus of I28E created a new analog named AM-8145 with improved selectivity for Nav 1.4 (300-fold) and Na 1.5 (6000-fold) and maintained potency for $\mathrm{Na}_{V}$ 1.7. Similar results were found for the addition of CyA at the N-terminus of I28E (analog AM-0422) and substitutions in the other selected positions. These properties were not present in the wild-type JZTX-V containing the Pra additions, but only in the I28E mutant. In summary, the optimization of JZTX-V toward a more selective $\mathrm{Na}_{V} 1.7$ inhibitor was possible through the extension of the $\mathrm{N}$-terminal with neutral or hydrophobic residues containing side-chains and addition of negative charge at the C-terminal. Interestingly, JZTX$\mathrm{V}$ share similar key residues with ProTx-II essential for the inhibition of $\mathrm{Na}_{\mathrm{V}} 1.7$, as for W5, M6, R26, and W24 in JZTX$\mathrm{V}$, and selectivity optimization for $\mathrm{Na}_{\mathrm{V}} 1.4$ and $\mathrm{Na}_{\mathrm{V}} 1.5$ through $\mathrm{N}$-terminal extensions.

\section{NaSpTx 7}

\section{JZTX-III}

The spider peptide JZTX-III ( $\beta / \kappa-T R T X-C g 1 a)$, also isolated from the tarantula Chilobrachys jingzhao, was first characterized as a potent inhibitor of TTX-R currents in rat cardiac myocytes with $\mathrm{IC}_{50}$ value of $380 \mathrm{nM}$ (Xiao et al., 2004), and later found to 


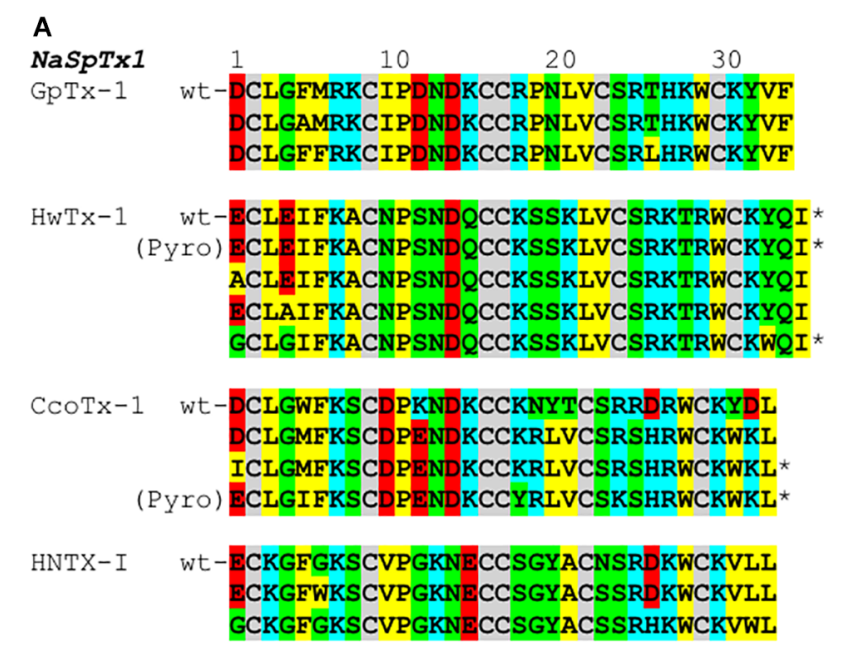

NaSpT×3

ProTX-II wt-YCQKWMWTCDSERKCCEGMVCRLWCKKKLW

YCQKWMWTCDSERKCCEGMVCRLWCKKKLW- $\mathrm{NHCH}_{3}$ GPYCQKWMQTCDSERKCCEGMVCRLWCKKKLL
JZTX-V Wt-YCQKWMWTCDSKRACCEGLRCKLWCRKII *
YCQKWMWTCDSKRACCEGLRCKLWCRKEI *
(Pra) YCQKWMWTCDSKRACCEGLRCKLWCRKEI *
( CYA) YCQKWMWTCDSKRACCEGLRCKLWCRKEI *
(Pra)

NaSpTx 7

JZTX-III Wt-DGECGGFWWKCGRGKPPCCKGYACSKTWGWCAVEAP

DGECGGFWWKCGEGKPPCCKGYACSKTWGWCAVEAP
B

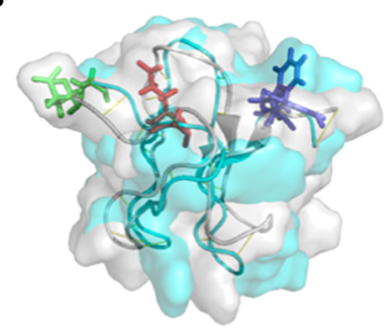

C

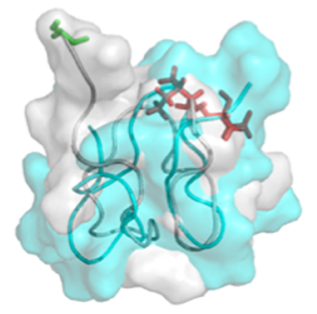

D

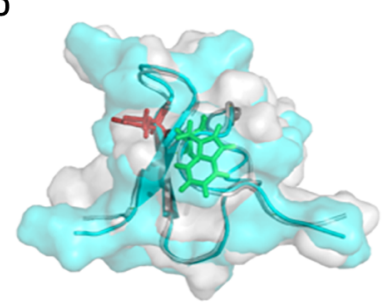

FIGURE 4 | SAR integration of spider ICK peptides. Primary and three-dimensional structures comparison of spider ICK peptides belonging to NaSpTx families 1 , 3 , and 7. (A) Primary structure alignment of wild-type peptides and respective optimized analogs showing enhancement of activity and/or selectivity of Nav channels. The amino acids residues are highlighted as follow: yellow for hydrophobic, red for acid, blue for basic, green for neutral, and gray for the cysteines. (B) Alignment of the three-dimensional structures of HwTx-IV (PDB 2m4x) (Minassian et al., 2013) colored in gray and gHwTx-IV (PDB 5tIr) (Agwa et al., 2017) colored in cyan. Structures are represented by cartoon and surface, and substitutions E1G, E4G and Y32W are represented by sticks colored in green, red, and blue, respectively. (C) Alignment of the three-dimensional structures of Pra-1 JZTX-V (PDB 6chc) colored in gray and AM-8145 (6cgw) colored in cyan (Moyer et al., 2018). Structures are represented by cartoon and surface, and the Pra-1 addition and substitution I28E are represented by sticks colored in green and red, respectively. (D) Alignment of the three-dimensional structures of HNTX-I (PDB 2mqf) colored in gray and the derived analog G7W/N24S (PDB 2mxo) colored in cyan (Klint et al., 2015a). Structures are represented by cartoon and surface, and substitutions G7W and N24S are represented by sticks colored in green and red, respectively. All three-dimensional structures alignments were performed in PyMOL (DeLano, 2002). Asterisk (*) denotes C-terminal amidation.

also inhibit $\mathrm{K}_{\mathrm{V}} 2.1$ channels (Yuan et al., 2007). The SAR of JZTXIII with $\mathrm{Na}_{V} 1.5$ channels using alanine substitutions revealed residues D1, E3 and W8, W9, W28, and W30 as key players for its Nav 1.5 inhibition (Rong et al., 2011) (Table 1 and Figure 2E). In addition, the substitution R13E enhanced the $\mathrm{Na}_{V} 1.5$ inhibition by 11 -fold (Figure 3F). Interestingly, JZTX-III does not inhibit the Nav1.7 channel, and this lack of affinity was associated to the residue $\mathrm{D} 816$ in the $\mathrm{Na}_{\mathrm{V}}$ 1.7. The substitution D816R (corresponding to the R800 in the $\mathrm{Na}_{\mathrm{V}} 1.5$ channel) enhanced significantly the inhibitory properties of JZTX-III for Nav 1.7.

\section{INTERACTIONS WITH THE CELL MEMBRANE}

Spider ICK peptides are known to interact with the lipids in the cell membrane. Early studies showed these peptides are water-soluble and bind to the aqueous-exposed extracellular surface of ion channels, and surprisingly reach the target by partitioning into the lipid membrane (Lee and MacKinnon, 2004). This strategy allows the peptide to reach the voltage-sensor and enhance high-affinity inhibition. The ability to bind to lipids seems exclusive of gating modifiers ICK peptides binding to site 4 of Nav channels (Smith et al., 2005). Furthermore, the Nav VSD s are affected by the cell membrane lipid composition, with changes from native lipids to sphingomyelin altering $\mathrm{G}-\mathrm{V}$ relations and affinity of ProTx-I for the domain II and domain IV S3-S4 loops (Milescu et al., 2009).

Detailed studies of these interactions using NMR revealed that ICK peptides can interact with the headgroup region of lipid membrane to induce a thinning of the bilayer (Mihailescu et al., 2014). In this interaction, many basic residues are positioned toward the aqueous phase, the $\mathrm{W}$ residues adopt an interfacial position and hydrophobic residues are in direct contact with the membrane. The SAR of ProTx-II, membrane lipids and Nav1.7 confirmed the previous observations, and revealed the ProTx-II 


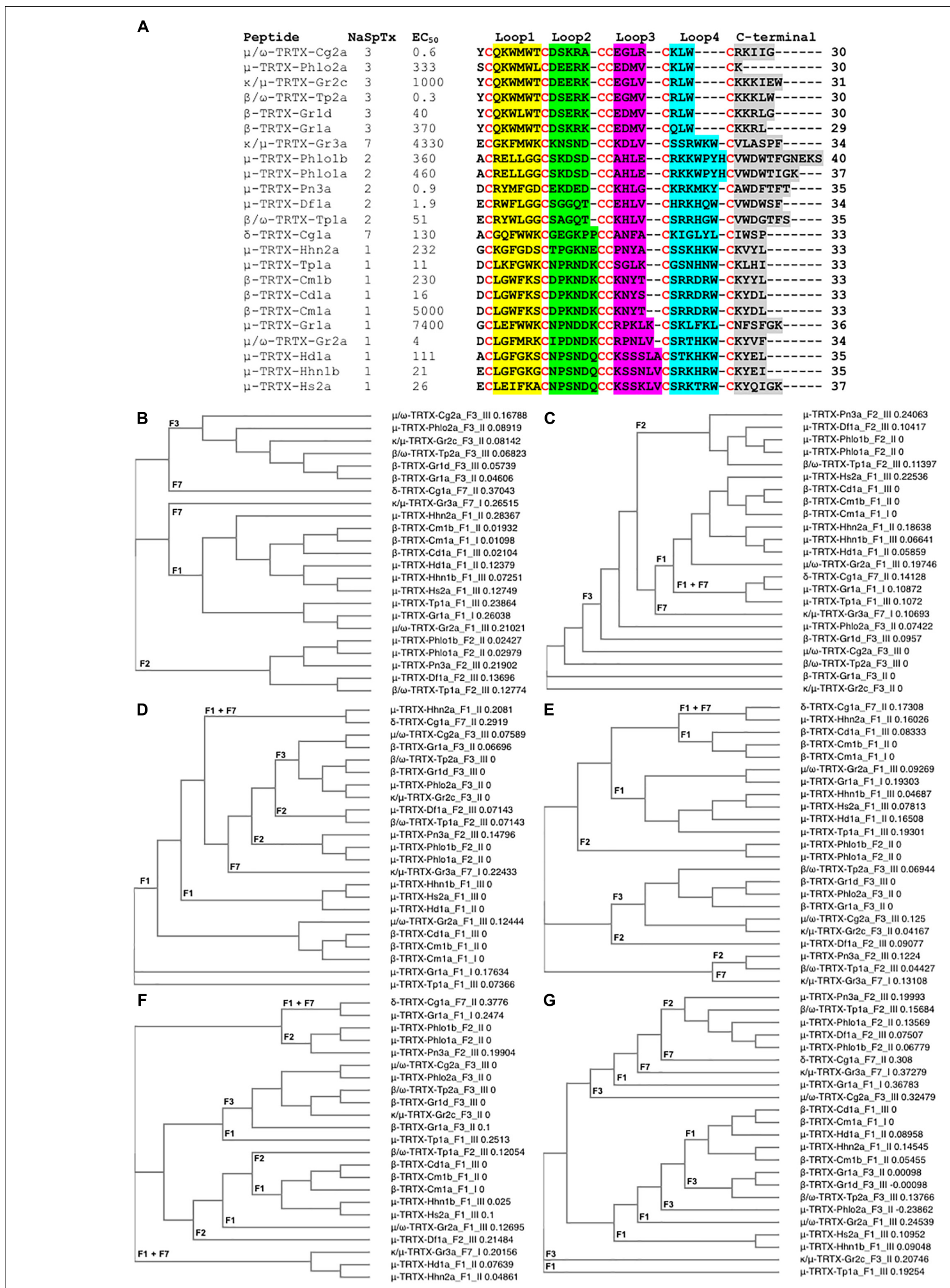

FIGURE 5 | Primary amino acids sequence alignment and phylogenetic analysis of NaSpTx peptides with described Nav1.7 modulatory activity. (A) Primary sequences alignment of members of NaSpTx1, 2, 3, and 7, with $\mathrm{EC}_{50}$ values represented in nM, Loops, 1, 2, 3, 4, and C-terminal shaded in yellow, green, pink,

(Continued) 
FIGURE 5 | Continued

blue and gray, respectively, and cysteines colored in red. (B) Phylogenetic analysis of the full primary sequences of NaSpTx NaSpTx1, 2, 3, and 7, followed by Loop 1 (C), Loop 2 (D), Loop 3 (E), Loop 4 (F), and C-terminals (G). For the phylogenetic trees, the peptides names are followed by their respective NaSpTx families represented by F1, F2, F3, and F7, and their Nav1.7 potency represented by I (EC $50>1 \mu \mathrm{M})$, II (EC 50 between 1 and $0.1 \mu \mathrm{M})$, and III (EC50 $<0.1 \mu \mathrm{M})$. These analyses were performed using Clustal Omega (Sievers et al., 2011) and Simple Phylogeny (Saitou and Nei, 1987). The loops were flanked by cysteine for these analyses.

analog E17K had increased on-rate for $\mathrm{Na}_{\mathrm{V}} 1.7$ compared to wildtype, but had no changes in the $\mathrm{Na}_{\mathrm{V}} 1.7$ potency (Henriques et al., 2016) (Table $\mathbf{1}$ and Figure 2F). Interestingly, all mutations at residues $\mathrm{W}$ to $\mathrm{Y}$ and $\mathrm{K}$ to $\mathrm{R}$ lead to a lower affinity for cell membranes. Changes in the structure of ICK peptides can also occur in the presence of lipids. This was observed in structural studies of $\omega$-Aga-IVA (a Ca channel inhibitor ICK peptide) that undergoes changes in the presence of micelles (Ryu et al., 2017). More specifically, the C-terminal tail of $\omega$-Aga-IVA assume a $\beta$-turn like conformation which is disordered in water.

The HwTx-IV mutant E1G/E4G/F6W/Y30W had increased affinity for the lipid membrane and higher potency for the inhibition of hNa 1.7 compared to wild-type (Agwa et al., 2017) (Table 1 and Figure 2G). Interestingly, the introduction of a PyroE in the N-terminal for HwTx-IV didn't alter the membrane interactions and potency for $\mathrm{Na}_{V}$ 1.7. This confirmed previous observations for the naturally occurring PyroE-HwTx-IV that binds irreversibly to the $\mathrm{Na}_{V}$ channel voltage-sensor but at same potency of wild-type HwTx-IV (Rong et al., 2013). Although membrane interactions are key for the high affinity binding of ICK peptides over ion channels, such interactions have to-date had little influence on the selectivity for the $\mathrm{Na}_{V}$ channel family (Agwa et al., 2018).

\section{BINDING SITES ON Nav CHANNELS}

The binding sites of spider ICK peptides at mammalian $\mathrm{Na}_{\mathrm{V}}$ channels are starting to be characterized (Cardoso and Lewis, 2017). These gating-modifier toxins are known for their ability to change the voltage-dependence of activation and inactivation of $\mathrm{Na}_{V}$ channels to either inhibit or activate $\mathrm{Na}^{+}$currents. Overall, they have preference for binding to the site 4 in domain II of the $\mathrm{Na}_{V}$ channel to trap the voltage-sensor and inhibit $\mathrm{Na}^{2+}$ currents, and to site 3 in domain IV to slow channel inactivation and maintain $\mathrm{Na}^{2+}$ currents. These sites have been reported for a range of spider ICK peptides, including HwTx-IV, ProTx-I, ProTx-II, PaurTx3, CcoTx-1, Hd1a and Dfla that inhibit $\mathrm{Na}_{\mathrm{V}}$ channels (Bosmans et al., 2008; Xiao et al., 2008a; Klint et al., 2015b; Shcherbatko et al., 2016; Cardoso et al., 2017) and to SGTx1 and Hmla that slow $\mathrm{Na}_{V}$ channels inactivation (Bosmans et al., 2008; Osteen et al., 2016).

The crystal structure of the related double-ICK spider peptide Dcla bound to the $\mathrm{Na}_{V} \mathrm{PaS}$ was determined (Shen et al., 2018). Dcla binds to a cleft between the VSD II and the pore loop of domain III of the $\mathrm{Na}_{V} \mathrm{PaS}$ and induce minimal changes in the channel structure. On the other hand, the Dcla peptide undergoes considerable rearrangement to achieve its fit into the cleft. This information brings new insights into the docking and binding of other ICK peptides into the mammalian channel, which although widely reported to bind to domain II and domain IV S3-S4 loops of mammalian $\mathrm{Na}_{\mathrm{V}}$ channels, could also be interacting with other proximal regions of the channel to reaches its final fit and maximal activities.

More recently, the cryo-EM structure of the human $\mathrm{Na}_{\mathrm{V}} 1.7$ channel was described in association of the auxiliary $\beta 1$ and $\beta 2$ subunits (Figures 1A,B) and the spider peptides HwTx-IV and ProTx-II (Shen et al., 2019). This study confirmed previous observations that HwTx-IV binds to the voltage-sensor of DII site 4 (Xiao et al., 2008a, 2010) and that ProTx-II binds to the voltage-sensors of DII and DIII sites 4 and 3 (Bosmans et al., 2008; Xiao et al., 2010). The cryo-EM structure of a chimeric hNa $1.7-$ $\mathrm{Na}_{\mathrm{V}} \mathrm{Ab}$ channel bound to ProTx-II was also described recently (Figure 1C) (Xu et al., 2019), which confirmed ProTx-II binds to the voltage-sensor of DII site 4 of the $\mathrm{Na}_{V} 1.7$ channel (Xiao et al., 2010). Although the determination of the three-dimensional structure of spider ICK peptides using X-ray or NMR are essential for the understanding of the SAR of these peptides, additional conformational changes are likely to happen for most of these ICK peptides when they achieved their final bound conformation with the $\mathrm{Na}_{V}$ channel and associated membrane lipids.

\section{SAR INTEGRATION OF SPIDER ICK PEPTIDES}

Prevalent features in the SAR of spider ICK peptides and $\mathrm{Na}_{\mathrm{V}}$ channels have been disclosed (Figure 4A and Table 1). Amongst the remarkable positions associated to $\mathrm{Na}_{V}$ activity in the $\mathrm{NaSpTx}$ 1 toxins are the residues 5 and 6 , which are typically occupied by the hydrophobic residue phenylalanine. Similarly, NaSpTx 3 toxins typically have positions 5 and 6 occupied by hydrophobic residues $\mathrm{W}$ and $\mathrm{M}$, which are also critical for $\mathrm{Na}_{V}$ inhibitory activity. Another interesting aspect of these SAR studies is apparent in optimized analogs with increased $\mathrm{Na}_{V}$ potency and selectivity that often display a decrease in negative charge at the $\mathrm{N}$-terminal compared to wild-type toxins. This is supported by the introduction of Pyro, Pra, CyA and the residues $G$ and P at the $\mathrm{N}$-terminal in optimized peptides belonging to NaSpTx families 1 and 3 (Rong et al., 2013; Shcherbatko et al., 2016; Flinspach et al., 2017; Moyer et al., 2018), and for the substitutions E1A, E1G and D1I in optimized peptides from NaSpTx 1 (Minassian et al., 2013; Revell et al., 2013; Shcherbatko et al., 2016; Rahnama et al., 2017; Zhang et al., 2018b). A decrease in negative charge at the N-terminal also enhances the lipid affinity of NaSpTx 1 toxins such as HwTx-IV (Henriques et al., 2016), suggesting these optimized peptides may have enhanced lipid binding in addition to any enhanced interactions directly with $\mathrm{Na}_{V}$ channels. This is 
relevant when alterations to $\mathrm{Na}_{\mathrm{V}}$ channel selectivity are sought, since enhanced lipid binding is unlikely to have less ability to influence $\mathrm{Na}_{V}$ channel subtype selectivity (Agwa et al., 2018).

Modifications in the C-terminal region that enhance inhibition of $\mathrm{Na}_{\mathrm{V}}$ channels often involve the introduction of positive charges (Figure $\mathbf{4 A}$ and Table $\mathbf{1}$ ). This is observed for ICK peptides belonging to NaSpTx 1 and 3, as for the C-terminal amidation of HwTx-IV and CcoTx-1, the introduction of a $\mathrm{NHCH}_{3}$ group in ProTx-II and the substitution D32K in CcoTx-1 (Minassian et al., 2013; Revell et al., 2013; Park et al., 2014; Shcherbatko et al., 2016). An increase in hydrophobicity in the C-terminal region also enhanced the $\mathrm{Na}_{V}$ inhibition properties of peptides in NaSpTx 1, especially Y33W in HwTxIV and Y31W in CcoTx-1 (Revell et al., 2013; Shcherbatko et al., 2016; Rahnama et al., 2017). Interestingly, the reduction in hydrophobicity and introduction of negative charges in the C-terminal of the NaSpTx 3 peptide JZTX-V led to a decrease in $\mathrm{Na}_{\mathrm{V}}$ affinity (analog I28E), but only at Nav1.4 (Moyer et al., 2018). A similar reduction in hydrophobicity in the $\mathrm{N}$-terminal region of the NaSpTx 1 analog F5A-GpTx-1 also decreased Nav1.4 inhibition (Murray et al., 2015). As our SAR knowledge of NaSpTx 1 and 3 deepens, we anticipate further opportunities to develop more selective and/or potent inhibitors are expected to emerge.

Structural changes in optimized molecules suggest gain of affinity can be associated with regions of the ICK peptide beyond those associated with the local effects of the substitution. For example, structural rearrangements are observed in the HwTxIV, JZTX-V and HNTX-I optimized analogs (Figures 4B-D) that alter the surface structure of wild-type vs. optimized peptide, with pronounced changes in the N-termini of JZVTX-V with the introduction of Pra (Figure 4C) and following the introduction of W7 in HNTX-I (Figure 4D). However, the three-dimensional structure of a NaSpTx inhibitor bound to a $\mathrm{Na}_{V}$ channel embedded in the cell membrane is still to be elucidated.

\section{THE PHYLOGENY OF NaSpTX TARGETING Nav1.7}

In addition to the SAR studies of the nine NaSpTx discussed above, a number of other NaSpTx display interesting $\mathrm{Na}_{\mathrm{V}} 1.7$ modulatory properties (Figure 5). To date, twenty-three other peptides belonging to NaSpTx 1, 2, 3, and 7 are described with potencies ranging from $0.3 \mathrm{nM}$ to $7.4 \mu \mathrm{M}$ for half-maximal activity for $\mathrm{Na}_{V} 1.7$ (Figure 5A). These include $\mu$-TRTXPhola, $\mu$-TRTX-Pholb and $\mu$-TRTX-Pho2a (Chow et al., 2015), $\kappa / \mu$-TRTX-Gr2c, $\kappa / \mu$-TRTX-Gr3a, $\mu$-TRTX-Gr1a, $\beta$-TRTXGr1d and $\beta$-TRTX-Gr1a (Redaelli et al., 2010), $\mu$-TRTX-Pn3a (Deuis et al., 2017), $\mu$-TRTX-Dfla (Cardoso et al., 2017), $\beta / \omega$-TRTX-Tp2a (Priest et al., 2007), $\delta$-TRTX-Cg1a (Xiao et al., 2005), $\mu$-TRTX-Tp1a (Cardoso et al., 2015), $\beta$-TRTX-Cm1b and $\beta$-TRTX-Cd1a (Bosmans et al., 2006; Sousa et al., 2017) and $\mu$-TRTX-Hd1a (Klint et al., 2015b). Amongst these, only

TABLE 2 | Therapeutical potential of NaSpTx peptides evaluated in pre-clinical rodent pain models.

\begin{tabular}{|c|c|c|c|c|c|}
\hline Peptide & $\begin{array}{l}\text { NaSpTx } \\
\text { family }\end{array}$ & Spider species & Preferred $\mathrm{Na}_{v}$ subtypes & $\begin{array}{l}\text { Therapeutic } \\
\text { potential }\end{array}$ & References \\
\hline $\begin{array}{l}\text { Gr1b (GsAFI) } \\
\text { Gr2c (GsAFII) }\end{array}$ & 3 & $\begin{array}{l}\text { Grammostola } \\
\text { rosea }\end{array}$ & $\begin{array}{l}\operatorname{Nav} 1.7>1.4>1.1>1.2 \\
\operatorname{Nav} 1.7>1.4>1.1\end{array}$ & $\begin{array}{l}\text { Acute and } \\
\text { inflammatory pain }\end{array}$ & Lampe, 1998 \\
\hline HwTx-IV & 1 & $\begin{array}{l}\text { Ornithoctonus } \\
\text { huwena }\end{array}$ & Nav $1.7>1.2>1.3>1.4$ & $\begin{array}{l}\text { Inflammatory and } \\
\text { SNI-induced } \\
\text { neuropathic pain }\end{array}$ & $\begin{array}{l}\text { Liu et al., } \\
2014 b\end{array}$ \\
\hline HnTx-IV & 1 & $\begin{array}{l}\text { Haplopelma } \\
\text { hainanum }\end{array}$ & $\mathrm{Na}_{\vee} 1.2>1.3>1.7$ & $\begin{array}{l}\text { SNI-induced } \\
\text { neuropathic and } \\
\text { formalin-induced } \\
\text { inflammatory pain. }\end{array}$ & $\begin{array}{l}\text { Liu et al., } \\
2014 a\end{array}$ \\
\hline ProTx-II & 3 & $\begin{array}{l}\text { Thrixopelma } \\
\text { Pruriens }\end{array}$ & $\mathrm{Na}_{V} 1.7>1.6>1.2>1.5>1.3>1.8$ & $\begin{array}{l}\text { Painful diabetic } \\
\text { neuropathy } \\
\text { Inflammatory pain }\end{array}$ & $\begin{array}{l}\text { Tanaka et al., } \\
\text { 2015; Flinspach } \\
\text { et al., } 2017\end{array}$ \\
\hline $\mathrm{HIla}$ & 7 & $\begin{array}{l}\text { Haplopelma } \\
\text { lividum }\end{array}$ & Nav1.8 & $\begin{array}{l}\text { Inflammatory and } \\
\text { neuropathic pain }\end{array}$ & $\begin{array}{l}\text { Meng et al., } \\
2016\end{array}$ \\
\hline Pn3a & 2 & $\begin{array}{l}\text { Pamphobeteus } \\
\text { nigricolor }\end{array}$ & Nav $1.7>1.1$ & $\begin{array}{l}\text { Inflammatory (with } \\
\text { opioid co- } \\
\text { administration) } \\
\text { and post-surgical } \\
\text { pain }\end{array}$ & $\begin{array}{l}\text { Deuis et al., } \\
\text { 2017; Mueller } \\
\text { et al., } 2019\end{array}$ \\
\hline Ca1a & Unknown & $\begin{array}{l}\text { Cyriopagopus } \\
\text { albostriatus }\end{array}$ & Nav $1.7>1.2>1.6>1.4>1.3$ & $\begin{array}{l}\text { Inflammatory and } \\
\text { thermal pain }\end{array}$ & $\begin{array}{l}\text { Zhang et al., } \\
2018 \mathrm{c}\end{array}$ \\
\hline $\mathrm{Ca} 2 \mathrm{a}$ & 1 & $\begin{array}{l}\text { Cyriopagopus } \\
\text { albostriatus }\end{array}$ & $\operatorname{Nav} 1.7>1.2>1.6>1.3$ & $\begin{array}{l}\text { Inflammatory and } \\
\text { thermal pain }\end{array}$ & $\begin{array}{l}\text { Zhang et al., } \\
2018 \text { a }\end{array}$ \\
\hline Cyriotoxin-1a & 1 & $\begin{array}{l}\text { Cyriopagopus } \\
\text { schioedtei }\end{array}$ & $\mathrm{Na}_{\mathrm{V}} 1.1>1.2>1.6>1.7>1.3$ & Thermal pain & $\begin{array}{l}\text { Goncalves } \\
\text { et al., } 2019\end{array}$ \\
\hline
\end{tabular}

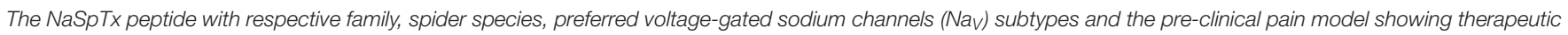

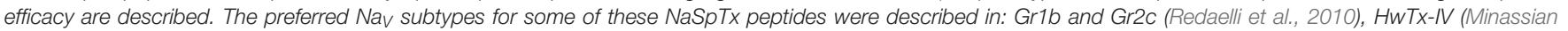
et al., 2013), HnTx-IV (Cai et al., 2015), and ProTx-Il (Middleton et al., 2002). 
$\delta$-TRTX-Cg1a activates $\mathrm{Na}_{V} 1.7$, while the other $\beta$ - and $\mu$-toxins are Nav1.7 inhibitors. Although detailed SAR studies on these toxins have not been described, their sequences similarities make a detailed analysis of their primary structure using phylogenetic approaches useful.

Not surprisingly, NaSpTxs targeting $\mathrm{Na}_{V} 1.7$ show independent phylogenetic origins and generate well defined clades for NaSpTx 1, 2, and 3, while NaSpTx 7, with only two representatives, clustered as outliers (Figure 5B). These results support previous findings underpinning the classification of the NaSpTx into 12 families (Klint et al., 2012). However, examining relatedness within loops revealed some interesting differences. For example, loop 1 showed similar clustering as the complete primary sequences except NaSpTx 7 clustered with NaSpTx 1 (Figure 5C), while loop 2 had more complex phylogenetic origins, with a primitive ancestor possibly arising from NaSpTx 1 (Figure 5D). Loop 3 also showed complex phylogenetic origins. It was divided into a major clade including the NaspTx1, two members of NaSpTx 2 and one member of NaSpTx 7, a second clade containing NaSpTx 3 and one member of the NaSpTx 2 as outlier, and a small clade including NaSpTx 2 and 7 (Figure 5E). These observations suggest a less purifying selection in loop 3, with NaSpTx 3 remaining the most conservative loop 3 group. The complexity of the phylogenetic origins of the loop 4 and C-terminal regions are considerably higher compared to loops 1, 2, and 3 (Figures 5F,G). Loop 4 diverged into four clades containing a mix of NaSpTx members, and again the NaSpTx 3 clustered further in a single clade, but now with one member of the NaSpTx 1 as an outlier. Finally, the C-terminal was diverged into three major clades that also contained a mix of $\mathrm{NaSpTx}$ members. Interestingly, the C-terminals of NaSpTx 2 clustered further into a single clade, indicating that a more conservative evolutionary pressure is occurring in this region of the NaSpTx 2 members.

Overall, we observed that the NaSpTx 1, 2 and 3 families have independent phylogenetic origins. For the NaSpTx 7, more representatives of this family are essential for an appropriated phylogenetic analysis. Remarkably, the loops 2, 3, and 4 forming the peptides in NaSpTx 3 are under a more conservative pressure compared to NaSpTx 1 and 2. This agrees with the SAR studies for ProTx-II ( $\beta / \omega$-TRTX-Tp2a), where optimization was possible only with modifications located in less conserved regions, the C-terminal and in the loop 1, while modifications in loops 2, 3 , and 4 were often deleterious for $\mathrm{Na}_{\mathrm{V}}$ inhibition (Priest et al., 2007; Smith et al., 2007; Schmalhofer et al., 2008; Park et al., 2014; Flinspach et al., 2017). For NaSpTx 1, loop 2 was more conserved and appears to be in agreement with the SAR of CcoTx1 ( $\beta$-TRTX-Cm1a) where modifications to loop 2 were not well tolerated, while loops 3, 4 and C-terminal tolerated multiple modification (Shcherbatko et al., 2016). Similarly, the C-terminal of NaSpTx 2 was also more conserved compared to the other NaSpTx families, but studies of SAR aiming to unravel the role this C-terminal region in $\mathrm{Na}_{V}$ inhibition are yet to be pursued. These patterns of evolutionary pressure provide clues to further explore the SAR of loops and C-terminal residues to that can help further expand the SAR of these $\mathrm{Na}_{V}$ channel toxins. However, a simple correlation between phylogenetic origins and potency at $\mathrm{Nav}$ channels remains to be established.

\section{PERSPECTIVES IN THERAPEUTIC DEVELOPMENT}

Advances in understanding disease mechanisms and associated novel therapeutic targets position spider ICK peptides as novel drug leads of sufficient size and pharmacophore complexity to restrict their in vivo distribution and limit off-target effects. To fully exploit this potential, a deeper understanding of ICK peptide SAR and bio-engineering is required to address specific therapeutic needs. This undoubtedly involves bio-activity and three-dimensional structural determinations for a detailed view of the SAR features. Pre-clinical studies in rodent pain models showing reversal of different types of pain have been reported for spider toxins, including several lacking published SAR data (Table 2).

The NaSpTx3 peptides Gr1b and Gr2c reversed acute and inflammatory pain intrathecally (Lampe, 1998), while ProTx-II and its optimized analog JNJ63955918 reversed neuropathic and inflammatory pain when administered intrathecally or locally (Tanaka et al., 2015; Flinspach et al., 2017). The NaSpTx1 peptides HwTx-IV and HNTX-IV also reversed neuropathic and inflammatory pains intraperitonially (Liu et al., 2014a,b), while ides $\mathrm{Ca} 2 \mathrm{a}$ and cyriotoxin-1a reversed inflammatory and thermal pain following intraperitoneal or intraplantar administration, respectively (Zhang et al., 2018a; Goncalves et al., 2019).

In contrast, the $\mathrm{NaSpTx} 2$ peptide Pn3a reversed inflammatory pain but only when co-administered with an intraperitoneal opioid (Deuis et al., 2017), despite reversing post-surgical pain following intraperitoneal or local administration (Mueller et al., 2019). Finally, the unclassified spider peptide Cala reversed inflammatory and thermal pain following intraperitoneal or intraplantar administrations, respectively (Zhang et al., 2018c). This review of the continuing advancements in the SAR of spider venom ICK peptides will hopefully facilitate efforts to optimize $\mathrm{Na}_{V}$ channel modulators for the treatment of complex channelopathies, including different forms of chronic pain.

\section{AUTHOR CONTRIBUTIONS}

FC performed the phylogenetic analysis, bibliography research, wrote the manuscript, and prepared the figures. RL provided scope, guidance and critically reviewed the manuscript.

\section{FUNDING}

The National Health and Medical Research Council of Australia APP1072113 provided research funding to RL that produced background to the review and APP1119056 provided a Fellowship to RL. 


\section{REFERENCES}

Abraham, N., and Lewis, R. J. (2018). Neuronal nicotinic acetylcholine receptor modulators from cone snails. Mar. Drugs 16:E208. doi: 10.3390/md16060208

Agwa, A. J., Lawrence, N., Deplazes, E., Cheneval, O., Chen, R., Craik, D. J., et al. (2017). Spider peptide toxin HwTx-IV engineered to bind to lipid membranes has an increased inhibitory potency at human voltage-gated sodium channel hNaV1.7. Biochim. Biophys. Acta 1859, 835-844. doi: 10.1016/j.bbamem.2017. 01.020

Agwa, A. J., Peigneur, S., Chow, C. Y., Lawrence, N., Craik, D. J., Tytgat, J., et al. (2018). Gating modifier toxins isolated from spider venom: modulation of voltage-gated sodium channels and the role of lipid membranes. J. Biol. Chem. 293, 9041-9052. doi: 10.1074/jbc.RA118.002553

Arnold, K., Bordoli, L., Kopp, J., and Schwede, T. (2006). The SWISSMODEL workspace: a web-based environment for protein structure homology modelling. Bioinformatics 22, 195-201. doi: 10.1093/bioinformatics/bti770

Bosmans, F., Martin-Eauclaire, M. F., and Swartz, K. J. (2008). Deconstructing voltage sensor function and pharmacology in sodium channels. Nature 456, 202-208. doi: 10.1038/nature07473

Bosmans, F., Rash, L., Zhu, S., Diochot, S., Lazdunski, M., Escoubas, P., et al. (2006). Four novel tarantula toxins as selective modulators of voltage-gated sodium channel subtypes. Mol. Pharmacol. 69, 419-429. doi: 10.1124/mol.105.015941

Cai, T., Luo, J., Meng, E., Ding, J., Liang, S., Wang, S., et al. (2015). Mapping the interaction site for the tarantula toxin hainantoxin-IV $(\beta$-TRTX-Hn2a) in the voltage sensor module of domain II of voltage-gated sodium channels. Peptides 68, 148-156. doi: 10.1016/j.peptides.2014.09.005

Cardoso, F. C., Dekan, Z., Rosengren, K. J., Erickson, A., Vetter, I., Deuis, J. R., et al. (2015). Identification and characterization of ProTx-III [ $\mu$-TRTX-Tpla], a new voltage-gated sodium channel inhibitor from the venom of the tarantula Thrixopelma pruriens. Mol. Pharmacol. 88, 291-303. doi: 10.1124/mol.115. 098178

Cardoso, F. C., Dekan, Z., Smith, J. J., Deuis, J. R., Vetter, I., Herzig, V., et al. (2017). Modulatory features of the novel spider toxin $\mu$-TRTX-Dfla isolated from the venom of the spider Davus fasciatus. Br. J. Pharmacol. 174, 2528-2544. doi: 10.1111/bph.13865

Cardoso, F. C., Hasan, M., Zhao, T., and Lewis, R. J. (2018). Toxins in pain. Curr. Opin. Support. Palliat. Care 12, 132-141. doi: 10.1097/SPC.0000000000000335

Cardoso, F. C., and Lewis, R. J. (2017). Sodium channels and pain: from toxins to therapies. Br. J. Pharmacol. 175, 2138-2157. doi: 10.1111/bph.13962

Chow, C. Y., Cristofori-Armstrong, B., Undheim, E. A., King, G. F., and Rash, L. D. (2015). Three peptide modulators of the human voltage-gated sodium channel 1.7, an important analgesic target, from the venom of an Australian tarantula. Toxins 7, 2494-2513. doi: 10.3390/toxins7072494

Cox, J. J., Reimann, F., Nicholas, A. K., Thornton, G., Roberts, E., Springell, K., et al. (2006). An SCN9A channelopathy causes congenital inability to experience pain. Nature 444, 894-898. doi: 10.1038/nature05413

DeLano, W. L. (2002). The PyMOL Molecular Graphics System, Version 1.5.0.4 Schrödinger. Scotts Valley, CA: LLC.

Deng, M., Luo, X., Jiang, L., Chen, H., Wang, J., He, H., et al. (2013). Synthesis and biological characterization of synthetic analogs of HuwentoxinIV ( $\mu$-theraphotoxin-Hh2a), a neuronal tetrodotoxin-sensitive sodium channel inhibitor. Toxicon 71, 57-65. doi: 10.1016/j.toxicon.2013.05.015

Deuis, J. R., Dekan, Z., Wingerd, J. S., Smith, J. J., Munasinghe, N. R., Bhola, R. F., et al. (2017). Pharmacological characterisation of the highly NaV1.7 selective spider venom peptide pN3a. Sci. Rep. 7:40883. doi: 10.1038/srep40883

Diochot, S., Drici, M. D., Moinier, D., Fink, M., and Lazdunski, M. (1999). Effects of phrixotoxins on the KV4 family of potassium channels and implications for the role of Ito1 in cardiac electrogenesis. Br. J. Pharmacol. 126, 251-263. doi: 10.1038/sj.bjp.0702283

Dongol, Y., Dhananjaya, B. L., Shrestha, R. K., and Aryal, G. (2016). Wasp venom toxins as a potential therapeutic agent. Protein Pept. Lett. 23, 688-698. doi: $10.2174 / 0929866523666160511151039$

Drenth, J. P., and Waxman, S. G. (2007). Mutations in sodium-channel gene SCN9A cause a spectrum of human genetic pain disorders. J. Clin. Invest. 117, 3603-3609. doi: 10.1172/JCI33297

Faber, C. G., Lauria, G., Merkies, I. S., Cheng, X., Han, C., Ahn, H. S., et al. (2012). Gain-of-function NaV1.8 mutations in painful neuropathy. Proc. Natl. Acad. Sci. U.S.A. 109, 19444-19449. doi: 10.1073/pnas.1216080109
Flinspach, M., Xu, Q., Piekarz, A. D., Fellows, R., Hagan, R., Gibbs, A., et al. (2017). Insensitivity to pain induced by a potent selective closed-state NaV1.7 inhibitor. Sci. Rep. 7:39662. doi: 10.1038/srep39662

Goncalves, T. C., Benoit, E., Kurz, M., Lucarain, L., Fouconnier, S., Combemale, S., et al. (2019). From identification to functional characterization of cyriotoxin-1a, an antinociceptive toxin from Cyriopagopus schioedtei spider. Br. J. Pharmacol. doi: 10.1111/bph.14628 [Epub ahead of print].

Grasso, G., Landi, A., and Alafaci, C. (2016). A novel pathophysiological mechanism contributing to trigeminal neuralgia. Mol. Med. 22, 452-454. doi: 10.2119/molmed.2016.00172

Henriques, S. T., Deplazes, E., Lawrence, N., Cheneval, O., Chaousis, S., Inserra, M., et al. (2016). Interaction of tarantula venom peptide ProTx-II with lipid membranes is a prerequisite for its inhibition of human voltage-gated sodium channel NaV1.7. J. Biol. Chem. 291, 17049-17065. doi: 10.1074/jbc.M116. 729095

Huang, J., Han, C., Estacion, M., Vasylyev, D., Hoeijmakers, J. G., Gerrits, M. M., et al. (2014). Gain-of-function mutations in sodium channel NaV1.9 in painful neuropathy. Brain 137, 1627-1642. doi: 10.1093/brain/awu079

Kessler, P., Marchot, P., Silva, M., and Servent, D. (2017). The three-finger toxin fold: a multifunctional structural scaffold able to modulate cholinergic functions. J. Neurochem. 142(Suppl. 2), 7-18. doi: 10.1111/jnc.13975

Klein-Weigel, P. F., Volz, T. S., and Richter, J. G. (2018). Erythromelalgia. Vasa 47, 91-97. doi: 10.1024/0301-1526/a000675

Klint, J. K., Chin, Y. K., and Mobli, M. (2015a). Rational engineering defines a molecular switch that is essential for activity of spider-venom peptides against the analgesics target NaV1.7. Mol. Pharmacol. 88, 1002-1010. doi: 10.1124/mol. 115.100784

Klint, J. K., Smith, J. J., Vetter, I., Rupasinghe, D. B., Er, S. Y., Senff, S., et al. (2015b). Seven novel modulators of the analgesic target NaV1.7 uncovered using a high-throughput venom-based discovery approach. Br. J. Pharmacol. 172, 2445-2458. doi: 10.1111/bph.13081

Klint, J. K., Senff, S., Rupasinghe, D. B., Er, S. Y., Herzig, V., Nicholson, G. M., et al. (2012). Spider-venom peptides that target voltage-gated sodium channels: pharmacological tools and potential therapeutic leads. Toxicon 60, 478-491. doi: 10.1016/j.toxicon.2012.04.337

Lampe, R. A. (1998). Analgesic peptides from venom of Grammostola spatulata and use thereof. U.S. Patent No 58,078,21A.

Lee, S. Y., and MacKinnon, R. (2004). A membrane-access mechanism of ion channel inhibition by voltage sensor toxins from spider venom. Nature 430, 232-235. doi: 10.1038/nature02632

Li, D., Xiao, Y., Xu, X., Xiong, X., Lu, S., Liu, Z., et al. (2004). Structure-activity relationships of hainantoxin-IV and structure determination of active and inactive sodium channel blockers. J. Biol. Chem. 279, 37734-37740. doi: 10. 1074/jbc.M405765200

Liao, Z., Yuan, C., Deng, M., Li, J., Chen, J., Yang, Y., et al. (2006). Solution structure and functional characterization of jingzhaotoxin-XI: a novel gating modifier of both potassium and sodium channels. Biochemistry 45, 15591-15600. doi: 10.1021/bi061457+

Liu, Y., Tang, J., Zhang, Y., Xun, X., Tang, D., Peng, D., et al. (2014a). Synthesis and analgesic effects of $\mu$-TRTX-Hhnlb on models of inflammatory and neuropathic pain. Toxins 6, 2363-2378. doi: 10.3390/toxins6082363

Liu, Y., Wu, Z., Tang, D., Xun, X., Liu, L., Li, X., et al. (2014b). Analgesic effects of Huwentoxin-IV on animal models of inflammatory and neuropathic pain. Protein Pept. Lett. 21, 153-158.

Liu, Z., Cai, T., Zhu, Q., Deng, M., Li, J., Zhou, X., et al. (2013). Structure and function of hainantoxin-III, a selective antagonist of neuronal tetrodotoxinsensitive voltage-gated sodium channels isolated from the Chinese bird spider Ornithoctonus hainana. J. Biol. Chem. 288, 20392-20403. doi: 10.1074/jbc. M112.426627

Luiz, A. P., and Wood, J. N. (2016). Sodium channels in pain and cancer: new therapeutic opportunities. Adv. Pharmacol. 75, 153-178. doi: 10.1016/bs.apha. 2015.12.006

Meisler, M. H., and Kearney, J. A. (2005). Sodium channel mutations in epilepsy and other neurological disorders. J. Clin. Invest. 115, 2010-2017. doi: 10.1172/ JCI25466

Meng, P., Huang, H., Wang, G., Yang, S., Lu, Q., Liu, J., et al. (2016). A novel toxin from Haplopelma lividum selectively inhibits the NaV1.8 channel and possesses potent analgesic efficacy. Toxins 9:7. doi: 10.3390/toxins9010007 
Middleton, R. E., Warren, V. A., Kraus, R. L., Hwang, J. C., Liu, C. J., Dai, G., et al. (2002). Two tarantula peptides inhibit activation of multiple sodium channels. Biochemistry 41, 14734-14747. doi: 10.1021/bi026546a

Mihailescu, M., Krepkiy, D., Milescu, M., Gawrisch, K., Swartz, K. J., and White, S. (2014). Structural interactions of a voltage sensor toxin with lipid membranes. Proc. Natl. Acad. Sci. U.S.A. 111, E5463-E5470. doi: 10.1073/pnas.1415324111

Milescu, M., Bosmans, F., Lee, S., Alabi, A. A., Kim, J. I., and Swartz, K. J. (2009). Interactions between lipids and voltage sensor paddles detected with tarantula toxins. Nat. Struct. Mol. Biol. 16, 1080-1085. doi: 10.1038/nsmb.1679

Minassian, N. A., Gibbs, A., Shih, A. Y., Liu, Y., Neff, R. A., Sutton, S. W., et al. (2013). Analysis of the structural and molecular basis of voltage-sensitive sodium channel inhibition by the spider toxin huwentoxin-IV ( $\mu$-TRTXHh2a). J. Biol. Chem. 288, 22707-22720. doi: 10.1074/jbc.M113.461392

Moyer, B. D., Murray, J. K., Ligutti, J., Andrews, K., Favreau, P., Jordan, J. B., et al. (2018). Pharmacological characterization of potent and selective NaV1.7 inhibitors engineered from Chilobrachys jingzhao tarantula venom peptide JzTx-V. PLoS One 13:e0196791. doi: 10.1371/journal.pone.0196791

Mueller, A., Starobova, H., Morgan, M., Dekan, Z., Cheneval, O., Schroeder, C. I., et al. (2019). Anti-allodynic effects of the selective NaV1.7 inhibitor Pn3a in a mouse model of acute post-surgical pain: evidence for analgesic synergy with opioids and baclofen. Pain [Epub ahead of print]. doi: 10.1097/j.pain. 0000000000001567

Murray, J. K., Ligutti, J., Liu, D., Zou, A., Poppe, L., Li, H., et al. (2015). Engineering potent and selective analogues of $\mathrm{GpTx}-1$, a tarantula venom peptide antagonist of the NaV1.7 sodium channel. J. Med. Chem. 58, 2299-2314. doi: 10.1021/ jm $501765 \mathrm{v}$

Murray, J. K., Long, J., Zou, A., Ligutti, J., Andrews, K. L., Poppe, L., et al. (2016). Single residue substitutions that confer voltage-gated sodium ion channel subtype selectivity in the NaV1.7 inhibitory peptide GpTx-1. J. Med. Chem. 59, 2704-2717. doi: 10.1021/acs.jmedchem.5b01947

Ono, S., Kimura, T., and Kubo, T. (2011). Characterization of voltagedependent calcium channel blocking peptides from the venom of the tarantula Grammostola rosea. Toxicon 58, 265-276. doi: 10.1016/j.toxicon.2011.06.006

Osteen, J. D., Herzig, V., Gilchrist, J., Emrick, J. J., Zhang, C., Wang, X., et al. (2016). Selective spider toxins reveal a role for the NaV1.1 channel in mechanical pain. Nature 534, 494-499. doi: 10.1038/nature17976

Park, J. H., Carlin, K. P., Wu, G., Ilyin, V. I., Musza, L. L., Blake, P. R., et al. (2014). Studies examining the relationship between the chemical structure of protoxin II and its activity on voltage gated sodium channels. J. Med. Chem. 57, 6623-6631. doi: 10.1021/jm500687u

Peng, K., Shu, Q., Liu, Z., and Liang, S. (2002). Function and solution structure of huwentoxin-IV, a potent neuronal tetrodotoxin (TTX)-sensitive sodium channel antagonist from Chinese bird spider Selenocosmia huwena. J. Biol. Chem. 277, 47564-47571. doi: 10.1074/jbc.M204063200

Phatarakijnirund, V., Mumm, S., Mcalister, W. H., Novack, D. V., Wenkert, D., Clements, K. L., et al. (2016). Congenital insensitivity to pain: fracturing without apparent skeletal pathobiology caused by an autosomal dominant, second mutation in SCN11A encoding voltage-gated sodium channel 1.9. Bone 84, 289-298. doi: 10.1016/j.bone.2015.11.022

Platnick, N. I. (2014). The World Spider Catalog, version 15. New York, NY: American Museum of Natural History.

Priest, B. T., Blumenthal, K. M., Smith, J. J., Warren, V. A., and Smith, M. M. (2007). ProTx-I and ProTx-II: gating modifiers of voltage-gated sodium channels. Toxicon 49, 194-201. doi: 10.1016/j.toxicon.2006.09.014

Rahnama, S., Deuis, J. R., Cardoso, F. C., Ramanujam, V., Lewis, R. J., Rash, L. D., et al. (2017). The structure, dynamics and selectivity profile of a NaV1.7 potency-optimised huwentoxin-IV variant. PLoS One 12:e0173551. doi: 10. 1371/journal.pone.0173551

Redaelli, E., Cassulini, R. R., Silva, D. F., Clement, H., Schiavon, E., Zamudio, F. Z., et al. (2010). Target promiscuity and heterogeneous effects of tarantula venom peptides affecting $\mathrm{Na}+$ and $\mathrm{K}+$ ion channels. J. Biol. Chem. 285, 4130-4142. doi: 10.1074/jbc.M109.054718

Revell, J. D., Lund, P. E., Linley, J. E., Metcalfe, J., Burmeister, N., Sridharan, S., et al. (2013). Potency optimization of Huwentoxin-IV on hNaV1.7: a neurotoxin TTX-S sodium-channel antagonist from the venom of the Chinese bird-eating spider Selenocosmia huwena. Peptides 44, 40-46. doi: 10.1016/j.peptides.2013. 03.011
Rong, M., Chen, J., Tao, H., Wu, Y., Jiang, P., Lu, M., et al. (2011). Molecular basis of the tarantula toxin jingzhaotoxin-III ( $\beta$-TRTX-Cj1 $\alpha)$ interacting with voltage sensors in sodium channel subtype Nav1.5. FASEB J. 25, 3177-3185. doi: 10.1096/fj.10-178848

Rong, M., Duan, Z., Chen, J., Li, J., Xiao, Y., and Liang, S. (2013). Native pyroglutamation of huwentoxin-IV: a post-translational modification that increases the trapping ability to the sodium channel. PLoS One 8:e65984. doi: 10.1371/journal.pone.0065984

Ryu, J. H., Jung, H. J., Konishi, S., Kim, H. H., Park, Z. Y., and Kim, J. I. (2017). Structure-activity relationships of $\omega$-Agatoxin IVA in lipid membranes. Biochem. Biophys. Res. Commun. 482, 170-175. doi: 10.1016/j.bbrc.2016.11.025

Saitou, N., and Nei, M. (1987). The neighbor-joining method: a new method for reconstructing phylogenetic trees. Mol. Biol. Evol. 4, 406-425.

Salvatierra, J., Castro, J., Erickson, A., Li, Q., Braz, J., Gilchrist, J., et al. (2018). NaV1.1 inhibition can reduce visceral hypersensitivity. JCI Insight 3:121000. doi: 10.1172/jci.insight. 121000

Schmalhofer, W. A., Calhoun, J., Burrows, R., Bailey, T., Kohler, M. G., Weinglass, A. B., et al. (2008). ProTx-II, a selective inhibitor of NaV1.7 sodium channels, blocks action potential propagation in nociceptors. Mol. Pharmacol. 74, 1476-1484. doi: 10.1124/mol.108.047670

Shcherbatko, A., Rossi, A., Foletti, D., Zhu, G., Bogin, O., Galindo Casas, M., et al. (2016). Engineering highly potent and selective microproteins against NaV1.7 sodium channel for treatment of pain. J. Biol. Chem. 291, 13974-13986. doi: 10.1074/jbc.M116.725978

Shen, H., Li, Z., Jiang, Y., Pan, X., Wu, J., Cristofori-Armstrong, B., et al. (2018). Structural basis for the modulation of voltage-gated sodium channels by animal toxins. Science 362:eaau2596. doi: 10.1126/science.aau2596

Shen, H., Liu, D., Wu, K., Lei, J., and Yan, N. (2019). Structures of human NaV1.7 channel in complex with auxiliary subunits and animal toxins. Science 363, 1303-1308. doi: 10.1126/science.aaw2493

Sievers, F., Wilm, A., Dineen, D., Gibson, T. J., Karplus, K., Li, W., et al. (2011). Fast, scalable generation of high-quality protein multiple sequence alignments using Clustal Omega. Mol. Syst. Biol. 7:539. doi: 10.1038/msb.2011.75

Sloan, G., Shillo, P., Selvarajah, D., Wu, J., Wilkinson, I. D., Tracey, I., et al. (2018). A new look at painful diabetic neuropathy. Diabetes Res. Clin. Pract. 144, 177-191. doi: 10.1016/j.diabres.2018.08.020

Smith, J. J., Alphy, S., Seibert, A. L., and Blumenthal, K. M. (2005). Differential phospholipid binding by site 3 and site 4 toxins. Implications for structural variability between voltage-sensitive sodium channel domains. J. Biol. Chem. 280, 11127-11133. doi: 10.1074/jbc.M412552200

Smith, J. J., Cummins, T. R., Alphy, S., and Blumenthal, K. M. (2007). Molecular interactions of the gating modifier toxin ProTx-II with NaV1.5: implied existence of a novel toxin binding site coupled to activation. J. Biol. Chem. 282, 12687-12697. doi: 10.1074/jbc.M610462200

Sousa, S. R., Wingerd, J. S., Brust, A., Bladen, C., Ragnarsson, L., Herzig, V., et al. (2017). Discovery and mode of action of a novel analgesic $\beta$-toxin from the African spider Ceratogyrus darlingi. PLoS One 12:e0182848. doi: 10.1371/ journal.pone.0182848

Szepetowski, P. (2018). Genetics of human epilepsies: continuing progress. Presse Med. 47, 218-226. doi: 10.1016/j.lpm.2017.10.020

Tanaka, K., Sekino, S., Ikegami, M., Ikeda, H., and Kamei, J. (2015). Antihyperalgesic effects of ProTx-II, a NaV1.7 antagonist, and A803467, a NaV1.8 antagonist, in diabetic mice. J. Exp. Pharmacol. 7, 11-16. doi: 10.2147/ JEP.S79973

Thompson, C. H., Kahlig, K. M., and George, A. L. Jr. (2011). SCN1A splice variants exhibit divergent sensitivity to commonly used antiepileptic drugs. Epilepsia 52, 1000-1009. doi: 10.1111/j.1528-1167.2011.03040.x

Wright, Z. V. F., Mccarthy, S., Dickman, R., Reyes, F. E., Sanchez-Martinez, S., Cryar, A., et al. (2017). The role of disulfide bond replacements in analogues of the tarantula toxin ProTx-II and their effects on inhibition of the voltagegated sodium ion channel NaV1.7. J. Am. Chem. Soc. 139, 13063-13075. doi: $10.1021 /$ jacs.7b06506

Xiao, Y., Bingham, J. P., Zhu, W., Moczydlowski, E., Liang, S., and Cummins, T. R. (2008a). Tarantula huwentoxin-IV inhibits neuronal sodium channels by binding to receptor site 4 and trapping the domain II voltage sensor in the closed configuration. J. Biol. Chem. 283, 27300-27313. doi: 10.1074/jbc. M708447200 
Xiao, Y., Luo, X., Kuang, F., Deng, M., Wang, M., Zeng, X., et al. (2008b). Synthesis and characterization of huwentoxin-IV, a neurotoxin inhibiting central neuronal sodium channels. Toxicon 51, 230-239.

Xiao, Y., Blumenthal, K., Jackson, J. O. II, Liang, S., and Cummins, T. R. (2010). The tarantula toxins ProTx-II and huwentoxin-IV differentially interact with human NaV1.7 voltage sensors to inhibit channel activation and inactivation. Mol. Pharmacol. 78, 1124-1134. doi: 10.1124/mol.110.066332

Xiao, Y., Tang, J., Hu, W., Xie, J., Maertens, C., Tytgat, J., et al. (2005). Jingzhaotoxin-I, a novel spider neurotoxin preferentially inhibiting cardiac sodium channel inactivation. J. Biol. Chem. 280, 12069-12076. doi: 10.1074/jbc. M411651200

Xiao, Y., Tang, J., Yang, Y., Wang, M., Hu, W., Xie, J., et al. (2004). JingzhaotoxinIII, a novel spider toxin inhibiting activation of voltage-gated sodium channel in rat cardiac myocytes. J. Biol. Chem. 279, 26220-26226. doi: 10.1074/jbc. M401387200

$\mathrm{Xu}$, H., Li, T., Rohou, A., Arthur, C. P., Tzakoniati, F., Wong, E., et al. (2019). Structural basis of NaV1.7 inhibition by a gatingmodifier spider toxin. Cell 176, 1238-1239. doi: 10.1016/j.cell.2019. 01.047

Yuan, C., Yang, S., Liao, Z., and Liang, S. (2007). Effects and mechanism of Chinese tarantula toxins on the KV2.1 potassium channels. Biochem. Biophys. Res. Commun. 352, 799-804. doi: 10.1016/j.bbrc.2006.11.086

Zhang, F., Liu, Y., Zhang, C., Li, J., Yang, Z., Gong, X., et al. (2015). Natural mutations change the affinity of $\mu$-theraphotoxin-Hhn2a to voltage-gated sodium channels. Toxicon 93, 24-30. doi: 10.1016/j.toxicon.2014.11.220
Zhang, Y., Peng, D., Huang, B., Yang, Q., Zhang, Q., Chen, M., et al. (2018a). Discovery of a novel NaV1.7 inhibitor from Cyriopagopus albostriatus venom with potent analgesic efficacy. Front. Pharmacol. 9:1158. doi: 10.3389/fphar. 2018.01158

Zhang, Y., Yang, Q., Zhang, Q., Peng, D., Chen, M., Liang, S., et al. (2018b). Engineering gain-of-function analogues of the spider venom peptide HNTXI, a potent blocker of the hNaV1.7 sodium channel. Toxins 10:E358. doi: 10 . 3390/toxins 10090358

Zhang, Y. X., Peng, D. Z., Zhang, Q. F., Huang, B., Yang, Q. C., Tang, D. F., et al. (2018c). $\mu$-TRTX-Ca1a: a novel neurotoxin from Cyriopagopus albostriatus with analgesic effects. Acta Pharmacol. Sin. doi: 10.1038/s41401-018-0181-9 [Epub ahead of print].

Ziegman, R., and Alewood, P. (2015). Bioactive components in fish venoms. Toxins 7, 1497-1531. doi: 10.3390/toxins7051497

Conflict of Interest Statement: The authors declare that the research was conducted in the absence of any commercial or financial relationships that could be construed as a potential conflict of interest.

Copyright (C) 2019 Cardoso and Lewis. This is an open-access article distributed under the terms of the Creative Commons Attribution License (CC BY). The use, distribution or reproduction in other forums is permitted, provided the original author(s) and the copyright owner(s) are credited and that the original publication in this journal is cited, in accordance with accepted academic practice. No use, distribution or reproduction is permitted which does not comply with these terms. 